\title{
New developments of the Extended Quadrature Method of Moments to solve Population Balance Equations
}

\author{
Maxime PIGOU, ${ }^{\mathrm{a}, \mathrm{b}, *}$, Jérôme MORCHAIN ${ }^{\mathrm{a}}$, Pascal FEDE ${ }^{\mathrm{b}}$, Marie-Isabelle PENET ${ }^{\mathrm{c}}$, \\ Geoffrey LARONZE \\ ${ }^{a}$ LISBP, Université de Toulouse, CNRS, INRA, INSA, Toulouse, France \\ ${ }^{b}$ Institut de Mécanique des Fluides de Toulouse - Université de Toulouse, CNRS-INPT-UPS, Toulouse, \\ France \\ ${ }^{c}$ Sanofi Chimie - CEBBD Biochemistry Vitry - 9 quai Jules Guesde 94400 Vitry-sur-Seine, France
}

\begin{abstract}
Population Balance Models have a wide range of applications in many industrial fields as they allow accounting for heterogeneity among properties which are crucial for some system modelling. They actually describe the evolution of a Number Density Function (NDF) using a Population Balance Equation (PBE). For instance, they are applied to gas-liquid columns or stirred reactors, aerosol technology, crystallisation processes, fine particles or biological systems. There is a significant interest for fast, stable and accurate numerical methods in order to solve for PBEs, a class of such methods actually does not solve directly the NDF but resolves their moments. These methods of moments, and in particular quadraturebased methods of moments, have been successfully applied to a variety of systems. Pointwise values of the NDF are sometimes required but are not directly accessible from the moments. To address these issues, the Extended Quadrature Method of Moments (EQMOM) has been developed in the past few years and approximates the NDF, from its moments, as a convex mixture of Kernel Density Functions (KDFs) of the same parametric family. In the present work EQMOM is further developed on two aspects. The main one is a significant improvement of the core iterative procedure of that method, the corresponding reduction of its computational cost is estimated to be between $80 \%$ and $85 \%$. The second aspect is an extension of EQMOM to two new KDFs used for the approximation, the Weibull and the Laplace kernels. All MATLAB source codes used for this article are provided with this article.
\end{abstract}

Keywords: Extended Quadrature Method of Moments (EQMOM), Quadrature Based Method of Moments (QBMM), Population Balance, Mathematical modelling, Gauss quadrature

\section{Introduction}

Population Balance Equations (PBEs) are particular formalisms that allows describing the evolution of properties among heterogeneous populations. They are used to track the size

${ }^{*}$ Corresponding author, Phone: +33561559275

Email address: maxime.pigou@insa-toulouse.fr (Maxime PIGOU) 
distribution of fine particles [1]; the bubble size distribution in gas-liquid stirred-tank reactors or bubble columns $[2,3]$; the crystal-size distribution in crystallizers or the distribution of biological cell properties in bioreactors [4, 5], among other examples.

A PBE describes the evolution and transport of a Number Density Function (NDF), under the influence of multiple processes which modify the tracked property distribution (e.g. erosion, dissolution, aggregation, breakage, coalescence, nucleation, adaptation, etc.).

One often requires low-cost numerical methods to solve PBEs, for instance when coupling with a flow solver (e.g. Computational Fluid Dynamics software). Monte-Carlo methods constitute a stochastic resolution of the population balance and can be applied to such PBE-CFD simulations [6]. Similarly, sectional methods allow direct numerical resolutions of the PBE through the discretisation of the property space $[7,8]$. They respectively require a high number of parcels or sections in order to reach high accuracy and are thus often discarded for large-scale simulations.

An interesting alternative approach lies in the field of methods of moments. A PBE, which describes the evolution of a NDF, is transformed in a set of equations which describes the evolution of the moments of that distribution. Moments are integral properties of NDFs, the first low order integer moments are related to the mean, variance, skewness and flatness of the statistical distributions described by NDFs. This approach then reduces the number of resolved variables to a finite set of NDF moments. It also comes with some difficulties when one must compute non-moment integral properties, or point-wise evaluations, of the distribution [9].

To tackle these issues, one can try to recover a NDF from a finite set of its moments. In most cases, this reverse problem has an infinite number of solutions and different approaches exist to identify one or an other out of them. Some methods that lead to continuous approximations are the Spline method [10], the Maximum-Entropy approach [9, 11, 12] or the Kernel Density Element Method (KDEM) [13].

More recently, the Extended Quadrature Method of Moments (EQMOM) was proposed as a new approach which is more stable than the previous ones, and yields either continuous or discrete NDFs depending on the moments $[1,14,15]$. EQMOM has been implemented in OpenFOAM [16] for the purpose of PBE-CFD coupling. The core of this method relies on an iterative procedure that is a computational bottleneck.

The current work focuses on EQMOM and develops a new core procedure whose computational cost is significantly lower than previous implementations by reducing both (i) the cost of each iteration and (ii) the total number of required iterations.

The previous core procedure [1] will be recalled before describing how it can be shifted toward the new -cheaper-approach. Both implementations will be compared in terms of computational cost (number of required floating-point operations) and run-time.

Multiple variations of EQMOM exist, the Gauss EQMOM [14, 17], Log-normal EQMOM [18] as well as Gamma and Beta EQMOM [15]. Two new variations, namely Laplace EQMOM and Weibull EQMOM, are proposed along with a unified formalism among all six variations.

The whole source code used to write this article (figures and data generation) is provided as supplementary data, as well as our implementations of EQMOM in the form of a MATLAB functions library [19]. 


\section{Quadrature Based Methods of Moments: QMOM and EQMOM}

\subsection{Definitions}

Let $\mathrm{d} \mu(\xi)$ be a positive measure, induced by a non-decreasing function $\mu(\xi)$ defined on a support $\Omega_{\xi}$. This measure is associated to a Number Density Function $n(\xi)$ such that $\mathrm{d} \mu(\xi)=n(\xi) \mathrm{d} \xi$. Let $\boldsymbol{m}_{N}$ be the vector of the first $N+1$ integer moments of this measure:

$$
\boldsymbol{m}_{N}=\left[\begin{array}{c}
m_{0} \\
m_{1} \\
\vdots \\
m_{N}
\end{array}\right], \quad m_{k}=\int_{\Omega_{\xi}} \xi^{k} n(\xi) \mathrm{d} \xi
$$

Three actual supports will be considered: (i) $\left.\Omega_{\xi}=\right]-\infty,+\infty\left[\right.$, (ii) $\left.\Omega_{\xi}=\right] 0,+\infty[$ and (iii) $\left.\Omega_{\xi}=\right] 0,1[$. For each support, one can define the associated realisable moment space, $\mathcal{M}_{N}\left(\Omega_{\xi}\right)$, as the set of all vectors of finite moments $\boldsymbol{m}_{N}$ induced by all possible positive measures defined on $\Omega_{\xi}$.

\subsection{Quadrature Method of Moments}

EQMOM is based on the Quadrature Method of Moments (QMOM) that was first introduced by McGraw [20]. It is used to approximate integral properties of a distribution where only a finite number of its moments is known. By making use of an even number of moments $2 P$, one can compute a Gauss quadrature rule characterised by its weights $\boldsymbol{w}_{P}=\left[w_{1}, \ldots, w_{P}\right]^{\top}$ and nodes $\boldsymbol{\xi}_{P}=\left[\xi_{1}, \ldots, \xi_{P}\right]^{\top}$ such that:

$$
\int_{\Omega_{\xi}} f(\xi) \mathrm{d} \mu(\xi)=\sum_{i=1}^{P} w_{i} f\left(\xi_{i}\right)
$$

holds true if $f(\xi)=\xi^{k}, \quad \forall k \in\{0, \ldots, 2 P-1\}$. Otherwise, this quadrature rule will produce an approximation of the integral property. The computation of the quadrature rule (i.e. the vectors $\boldsymbol{w}_{P}$ and $\boldsymbol{\xi}_{P}$ ) is of special interest for us, which is why its two main steps will be detailed.

Any positive measure $\mathrm{d} \mu(\xi)$ is associated with a sequence of monic polynomials (i.e. polynomial whose leading coefficient equals 1 ) denoted $\pi_{k}$-with $k$ the order of the polynomialsuch that:

$$
\int_{\Omega_{\xi}} \pi_{i}(\xi) \pi_{j}(\xi) \mathrm{d} \mu(\xi)=0, \quad \text { for } i \neq j
$$

These polynomials are said orthogonal with respect to the measure $\mathrm{d} \mu(\xi)$ and are defined by:

$$
\pi_{k}(\xi)=\frac{1}{c_{k}}\left|\begin{array}{ccccc}
m_{0} & m_{1} & \cdots & m_{k-1} & m_{k} \\
m_{1} & m_{2} & \cdots & m_{k} & m_{k+1} \\
\vdots & \vdots & \ddots & \vdots & \vdots \\
m_{k-1} & m_{k} & \cdots & m_{2 k-2} & m_{2 k-1} \\
1 & \xi & \cdots & \xi^{k-1} & \xi^{k}
\end{array}\right|
$$


with $c_{k}$ a constant chosen so that the leading coefficient (of order $k$ ) of $\pi_{k}$ equals 1 , hence making $\pi_{k}$ a monic polynomial.

It is known that monic orthogonal polynomials satisfy a three-term recurrence relation [21]:

$$
\pi_{k+1}(\xi)=\left(\xi-a_{k}\right) \pi_{k}(\xi)-b_{k} \pi_{k-1}(\xi)
$$

with $a_{k}$ and $b_{k}$ being the recurrence coefficients specific to the measure $\mathrm{d} \mu(\xi), \pi_{-1}(\xi)=0$ and $\pi_{0}(\xi)=1$.

Let $\boldsymbol{J}_{n}(\mathrm{~d} \mu)$ be the $n \times n$ Jacobi matrix associated to the measure $\mathrm{d} \mu$. This is a tridiagonal symmetric matrix defined as:

$$
\boldsymbol{J}_{n}(\mathrm{~d} \mu)=\left(\begin{array}{cccc}
a_{0} & \sqrt{b_{1}} & & 0 \\
\sqrt{b_{1}} & a_{1} & \ddots & \\
& \ddots & \ddots & \sqrt{b_{n-1}} \\
0 & & \sqrt{b_{n-1}} & a_{n-1}
\end{array}\right)
$$

The weights and nodes of the quadrature rule from Eq. (2) are given by spectral properties of $\boldsymbol{J}_{P}(\mathrm{~d} \mu)$. The nodes $\boldsymbol{\xi}_{P}$ of the rule are the eigenvalues of $\boldsymbol{J}_{P}(\mathrm{~d} \mu)$. The weights of the rule are given by:

$$
w_{i}=m_{0} v_{1, i}^{2}
$$

where $v_{1, i}$ is the first component of the normalised eigenvector belonging to the eigenvalue $\xi_{i}$. The computation of the quadrature rule (Eq. (2)) then relies on two steps:

1. The computation of the recurrence coefficients $\boldsymbol{a}_{P-1}=\left[a_{0}, \ldots, a_{P-1}\right]^{\top}$ and $\boldsymbol{b}_{P-1}=$ $\left[b_{1}, \ldots, b_{P-1}\right]^{\top}$.

2. The computation of the eigenvalues and the normalised eigenvectors of $\boldsymbol{J}_{P}(\mathrm{~d} \mu)$.

Multiple algorithms are available in the literature to compute the recurrence coefficients:

- The Quotient-Difference algorithm [22, 23]

- The Product-Difference algorithm [24]

- The Chebyshev algorithm [25]

The Chebyshev algorithm was found to be the stablest one of the three [1,25], its description is given in Appendix A.

\subsection{Extended Quadrature Method of Moments}

The QMOM method is well suited for the approximation of integral properties of the NDF, which is actually the main purpose of Gauss quadratures. However, in many applications such as evaporation [9] or dissolution [26] processes, point-wise values of the NDF $n(\xi)$ are required but not directly accessible from the moments. For that purpose, a method is needed to produce an approximation $\widetilde{n}(\xi)$ of the original distribution $n(\xi)$, by knowing only a finite set of its moments. 
In a sense, one can consider that the Gaussian quadrature computed with QMOM approximates $n(\xi)$ as a weighted sum of Dirac distributions:

$$
\widetilde{n}(\xi)=\sum_{i=1}^{P} w_{i} \delta\left(\xi, \xi_{i}\right)
$$

with the Dirac $\delta$ distribution defined by its sifting property

$$
\int_{-\infty}^{+\infty} f(\xi) \delta\left(\xi, \xi_{m}\right) \mathrm{d} \xi=f\left(\xi_{m}\right)
$$

For most applications, $n(\xi)$ is expected to be a continuous distribution whilst QMOM yields monodisperse or discrete polydisperse reconstructions of $n(\xi)$, with $\widetilde{n}(\xi)=0$ for all values of $\xi$ except some finite number of these values.

Many methods were suggested to tackle this problem and to propose a continuous reconstruction $\widetilde{n}(\xi)$ from a finite number of moments $\boldsymbol{m}_{N}$. Some of them are the Spline method [10], the Maximum-Entropy approach [11, 12, 9] or the Kernel Density Element Method [13]. Their properties will not be discussed here but one only underlines that they tend to be unstable, ill-conditionned, or have a high sensitivity to numerical parameters [10, 26, 27]. In particular, none of them can handle the case of a moment set which would be on the boundary of the realisable moment space $\boldsymbol{m}_{N} \in \partial \mathcal{M}_{N}\left(\Omega_{\xi}\right)$. Such a moment set is associated to a discrete (or degenerated) distribution and, in this specific case, the solution provided by $\mathrm{QMOM}$ is the only possible reconstruction.

Note that a failure - or instabilities- in a numerical method can compromise the integrity of large-scale simulations. For this reason, Chalons et al. [14], Yuan et al. [15] and Nguyen et al. [1] proposed a robust and stable method to tackle this reconstruction problem by handling both continuous approximations and discrete solutions. Their approach, the Extended Quadrature Method of Moments, approximates $n(\xi)$ as a convex mixture of Kernel Density Functions (KDFs) of the same parametric family:

$$
\widetilde{n}(\mu)=\sum_{i=1}^{P} w_{i} \delta_{\sigma}\left(\xi, \xi_{i}\right)
$$

with

- $w_{i}$ : the weight of the i-th node, $w_{i} \geq 0, \forall i \in\{1, \ldots, P\}$

- $\xi_{i}$ : the location parameter of the i-th node, $\xi_{i} \in \Omega_{\xi}, \forall i \in\{1, \ldots, P\}$

- $\delta_{\sigma}$ : a KDF chosen to perform the approximation, referred later to as the reconstruction kernel. $\sigma$ is the shape parameter of the approximation.

The computation of the weights $\boldsymbol{w}_{P}=\left[w_{1}, \ldots, w_{P}\right]^{\top}$, the nodes $\boldsymbol{\xi}_{P}=\left[\xi_{1}, \ldots, \xi_{P}\right]^{\top}$ and the shape parameter $\sigma$ from the moment set $\boldsymbol{m}_{2 P}$ is performed by the EQMOM momentinversion procedure. The improvement of this procedure constitutes the core of this article and is detailed in section 3 . 
Multiple standard normalized distribution functions can be used as the reconstruction kernel $\delta_{\sigma}$ (e.g. Gaussian, Log-normal, etc.). A list of them is given in Appendix B. All of these kernels degenerate into Dirac distribution if their shape parameters are sufficiently small:

$$
\lim _{\sigma \rightarrow 0} \delta_{\sigma}\left(\xi, \xi_{m}\right)=\delta\left(\xi, \xi_{m}\right)
$$

This allows EQMOM to perfectly handle the case of a moment set $\boldsymbol{m}_{2 P}$ being on the boundary of the realisable moment space $\partial \mathcal{M}_{2 P}\left(\Omega_{\xi}\right)$.

EQMOM can also be used to compute integral properties of the NDF with high accuracy. This comes with the introduction of nested quadratures. The main quadrature proposes the following approximation of integral terms:

$$
\int_{\Omega_{\xi}} f(\xi) n(\xi) \mathrm{d} \xi \approx \sum_{i=1}^{P} w_{i}\left[\int_{\Omega_{\xi}} f(\xi) \delta_{\sigma}\left(\xi, \xi_{i}\right) \mathrm{d} \xi\right]
$$

Moreover, a quadrature rule can be used to approximate the bracketed integral in Eq. (12). This will be the nested quadrature that actually depends on the kernel $\delta_{\sigma}\left(\xi, \xi_{m}\right)$. For instance, Gauss-Hermite quadratures can be used to approximate integrals over a Gaussian kernel (see Appendix B.1). Nested quadratures then give the following approximation:

$$
\int_{\Omega_{\xi}} f(\xi) n(\xi) \mathrm{d} \xi \approx \sum_{i=1}^{P} w_{i} \sum_{j=1}^{Q} \omega_{j} f\left(\xi_{i j}^{(\sigma)}\right)
$$

with $Q$ the order of the sub-quadrature, $\boldsymbol{\omega}_{Q}=\left[\omega_{1}, \ldots, \omega_{Q}\right]^{\top}$ the weights of the sub-quadrature, and $\xi_{i j}^{(\sigma)}$ the $j$-th node of the sub-quadrature, taking into account the location and shape parameters of the $i$-th main-quadrature node. These nested quadratures are detailed for all KDFs in Appendix B and Appendix C.

\section{Moment inversion procedure}

The EQMOM moment-inversion procedure comes with analytical solutions for some kernels in the case of low-order quadratures. The one-node analytical solutions are detailed for all kernels in Appendix B. When they exist, the two-nodes analytical solutions are implemented in MATLAB code (see supplementary data) but are not detailed in this article. The current section is focusing on the numerical procedure used to compute the reconstruction parameters in absence of an analytical solution.

The procedure proposed by Yuan et al. [15] and Nguyen et al. [1] is first recalled in section 3.1. The section 3.2 details how their approach can be shifted toward a new convergence criteria that will be applied to the specific cases of

- the Hamburger moment problem (section 3.3): NDF defined on the whole phase space $\left.\Omega_{\xi}=\right]-\infty,+\infty[$

- the Stieltjes moment problem (section 3.4): NDF defined on the positive phase space $\left.\Omega_{\xi}=\right] 0,+\infty[$ 
- the Hausdorff moment problem (section 3.5): NDF defined on the closed support $\left.\Omega_{\xi}=\right] 0,1[$

\subsection{Standard procedure}

Let $\boldsymbol{m}_{N}$ be the vector of the first $N+1$ integer moments of the measure $\mathrm{d} \mu(\xi)=n(\xi) \mathrm{d} \xi$, with $N=2 P$ an even integer:

$$
\boldsymbol{m}_{N}=\left[\begin{array}{c}
m_{0} \\
m_{1} \\
\vdots \\
m_{N}
\end{array}\right], \quad m_{k}=\int_{\Omega_{\xi}} \xi^{k} n(\xi) \mathrm{d} \xi
$$

The EQMOM moment-inversion procedure aims to identify the parameters $\sigma, \boldsymbol{w}_{P}=$ $\left[w_{1}, \ldots, w_{P}\right]^{\top}$ and $\boldsymbol{\xi}_{P}=\left[\xi_{1}, \ldots, \xi_{P}\right]^{\top}$ such that $\boldsymbol{m}_{N}=\widetilde{\boldsymbol{m}}_{N}$ with:

$$
\widetilde{\boldsymbol{m}}_{N}=\left[\begin{array}{c}
\widetilde{m}_{0} \\
\widetilde{m}_{1} \\
\vdots \\
\widetilde{m}_{N}
\end{array}\right], \quad \widetilde{m}_{k}=\int_{\Omega_{\xi}} \xi^{k} \widetilde{n}(\xi) \mathrm{d} \xi, \quad \widetilde{n}(\xi)=\sum_{i=1}^{P} w_{i} \delta_{\sigma}\left(\xi, \xi_{i}\right)
$$

For any value of $\sigma$, Yuan et al. [15] identified a procedure which leads to the parameters $\boldsymbol{w}_{P}$ and $\boldsymbol{\xi}_{P}$ such that $\boldsymbol{m}_{N-1}=\widetilde{\boldsymbol{m}}_{N-1}$. The EQMOM moment-inversion problem has then been reduced to solving a scalar non-linear equation by looking for a root of the function $D_{N}(\sigma)=m_{N}-\widetilde{m}_{N}(\sigma)$.

The approach developed by Yuan et al. [15] and then improved by Nguyen et al. [1] is based on the fact that, for the KDFs used in EQMOM, it is possible to write the following linear system:

$$
\widetilde{\boldsymbol{m}}_{n}=\boldsymbol{A}_{n}(\sigma) \cdot \boldsymbol{m}_{n}^{*}
$$

where $\boldsymbol{A}_{n}(\sigma)$ is a lower-triangular $(n+1) \times(n+1)$ matrix whose elements depend only on the chosen $\mathrm{KDF}$ and on the value $\sigma$, whereas $\boldsymbol{m}_{n}^{*}$ is defined as:

$$
\boldsymbol{m}_{n}^{*}=\left[\begin{array}{c}
m_{0}^{*} \\
m_{1}^{*} \\
\vdots \\
m_{n}^{*}
\end{array}\right], \quad m_{k}^{*}=\sum_{i=1}^{P} w_{i} \xi_{i}^{k}
$$

By their definition, the moments $\boldsymbol{m}_{n}^{*}$ correspond to the moments of a degenerated distribution (i.e. a finite sum of Dirac distributions), hence these moments will be referred as the degenerated moments of the approximation. Degenerated moments are defined in such a way that the vectors $\boldsymbol{w}_{P}$ and $\boldsymbol{\xi}_{P}$ can be computed from $\boldsymbol{m}_{2 P-1}^{*}$ using a Gauss Quadrature (see 2.2).

At this point, one has the basis required to compute the objective function $D_{N}(\sigma)$ and to search for its root. The computation of $D_{N}(\sigma)$ from a vector $\boldsymbol{m}_{N}$ is as follow (see also Fig. 1a): 
1. Compute $\boldsymbol{m}_{N-1}^{*}(\sigma)=\boldsymbol{A}_{N-1}^{-1}(\sigma) \cdot \boldsymbol{m}_{N-1}$.

2. Compute the recurrence coefficients $\boldsymbol{a}_{P-1}^{*}(\sigma)$ and $\boldsymbol{b}_{P-1}^{*}(\sigma)$ by applying the Chebyshev algorithm to $\boldsymbol{m}_{N-1}^{*}(\sigma)$.

3. Use the recurrence coefficients to compute the Gaussian quadrature rule $\boldsymbol{w}_{P}(\sigma)$ and $\boldsymbol{\xi}_{P}(\sigma)$.

4. Knowing the parameters $\sigma, \boldsymbol{w}_{P}(\sigma)$ and $\boldsymbol{\xi}_{P}(\sigma)$ of the reconstruction, compute $\widetilde{m}_{N}(\sigma)$, this can be done easily by:

- Computing the N-th order degenerated moment of the approximated NDF: $\bar{m}_{N}^{*}(\sigma)=$ $\sum_{i=1}^{P} w_{i}(\sigma) \xi_{i}(\sigma)^{N}$.

- Multiplying the last line of $\boldsymbol{A}_{N}(\sigma)$ and the vector of degenerated moments: $\widetilde{m}_{N}(\sigma)=$ $[0,0, \ldots, 1] \cdot \boldsymbol{A}_{N}(\sigma) \cdot\left[m_{0}^{*}(\sigma), \ldots, m_{N-1}^{*}(\sigma), \bar{m}_{N}^{*}(\sigma)\right]^{\top}$.

5. Compute $D_{N}(\sigma)=m_{N}-\widetilde{m}_{N}(\sigma)$.

For each compatible KDF, it is possible to use the low order moments to compute an upper bound $\sigma_{\max }$ so that the search of a root of $D_{N}$ is restricted to the interval $\sigma \in\left[0, \sigma_{\max }\right]$. Then a bounded non-linear equation solver such as Ridder's method can be applied to actually find the root of the function.

Two specific cases were discarded in the previous description of the method. First, it happens that the function $D_{N}$ does not admit any root, in such a case the procedure is switched toward the minimisation of this function in order to reduce the error on the last moment of the approximation.

Second, during the computation of $D_{N}(\sigma)$, one must compute degenerated moments from which the weights and nodes are extracted. If the degenerated moments $\boldsymbol{m}_{N-1}^{*}(\sigma)$ turn out not to be realisable on the support $\Omega_{\xi}$ of the NDF, the quadrature performed on this vector will lead to nodes outside $\Omega_{\xi}$, or even to negative/complex weights. Nguyen et al. [1] then suggest to check for the realisability of the degenerated moments, and if these are not realisable, to set $\widetilde{m}_{N}(\sigma)$ to a arbitrarily high value such as $10^{100}$. This will force the nonlinear equation solver to test a lower value of $\sigma$ in order to bring back the vector $\boldsymbol{m}_{N-1}^{*}(\sigma)$ within the realisable moment space. However note that this is only a numerical trick to converge toward the actual root, but $D_{N}(\sigma)$ is actually undefined as soon as $\boldsymbol{m}_{N-1}^{*}(\sigma)$ is not realisable.

\subsection{A new procedure based on moment realisability}

The reversible linear system linking the raw moments of the approximation $\widetilde{\boldsymbol{m}}_{N}$ to its degenerated moments $\boldsymbol{m}_{N}^{*}$ is such that a new objective function $D_{N}^{*}(\sigma)$-whose root is the same as that of $D_{N}(\sigma)$ - can be formulated. Its computation is as follow (see also Fig. 1b):

1. Compute $\boldsymbol{m}_{N}^{*}(\sigma)=\boldsymbol{A}_{N}^{-1}(\sigma) \cdot \boldsymbol{m}_{N}$.

2. Compute a quadrature on the vector $\boldsymbol{m}_{N-1}^{*}(\sigma)$ to obtain the vectors $\boldsymbol{w}_{P}(\sigma)$ and $\boldsymbol{\xi}_{P}(\sigma)$.

3. Compute $\bar{m}_{N}^{*}(\sigma)=\sum_{i=1}^{P} w_{i}(\sigma) \xi_{i}(\sigma)^{N}$.

4. Compute $D_{N}^{*}(\sigma)=m_{N}^{*}(\sigma)-\bar{m}_{N}^{*}(\sigma)$.

The benefit of this new objective function is that it only requires the matrix $\boldsymbol{A}_{N}^{-1}(\sigma)$ instead of both the matrix $\boldsymbol{A}_{N-1}^{-1}(\sigma)$ and the last line of $\boldsymbol{A}_{N}(\sigma)$. This only increases the clarity of the method, but has hardly no effect on its numerical cost. 
(a)

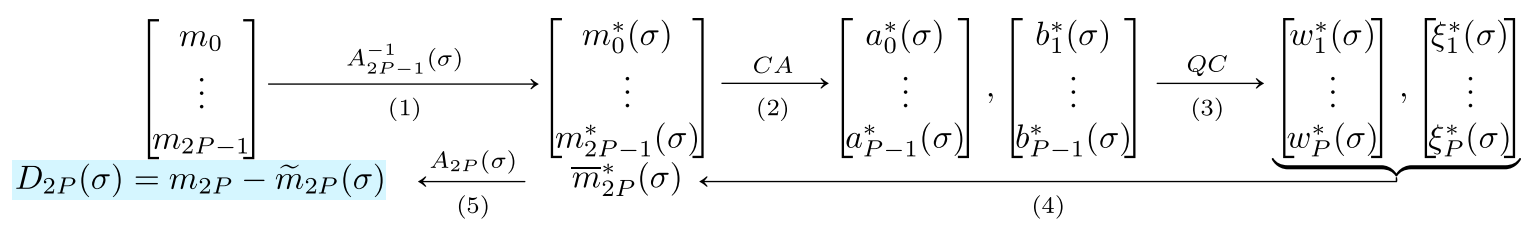

(b)

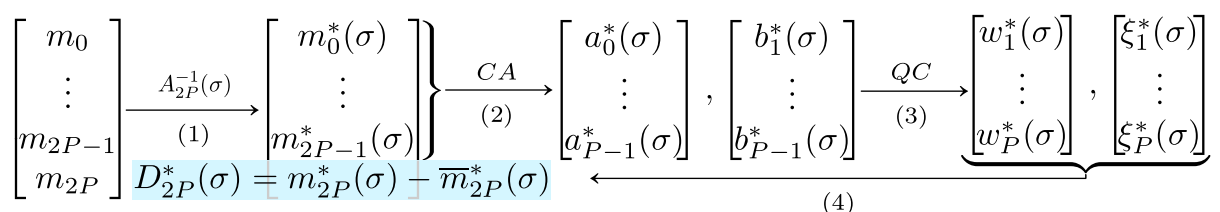

(c)

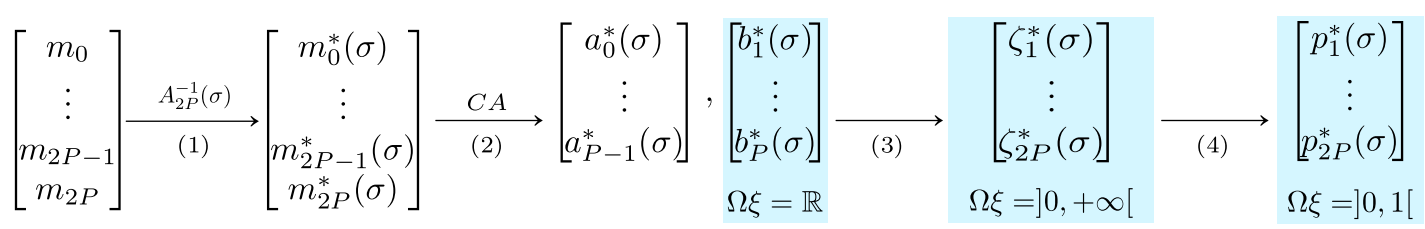

Figure 1: Comparison of the computation of convergence criteria based on (a) $D_{N}(\sigma),(\mathrm{b}) D_{N}^{*}(\sigma)$ and $(\mathrm{c})$ the realisability criteria of the support $\Omega_{\xi}$. CA: Chebyshev Algorithm. QC: Quadrature Computation. The convergence criteria are highlighted in light blue. Inspired by Fig. 1 from Nguyen et al. [1].

The point of this alternative approach is however to underline a crucial element for the new EQMOM implementation: we actually look for a value of $\sigma$ for which $m_{2 P}^{*}(\sigma)=\bar{m}_{2 P}^{*}(\sigma)$. This implies that, for this specific searched $\sigma$ value, the vector $\boldsymbol{m}_{2 P}^{*}(\sigma)$ reads

$$
\boldsymbol{m}_{2 P}^{*}(\sigma)=\left[\begin{array}{c}
\sum_{i=1}^{P} w_{i} \xi_{i}^{0} \\
\sum_{i=1}^{P} w_{i} \xi_{i}^{1} \\
\vdots \\
\sum_{i=1}^{P} w_{i} \xi_{i}^{2 P}
\end{array}\right]
$$

which is, by construction, the vector of the first $2 P+1$ moments of the sum of $P$ Dirac distributions. Under the condition $\xi_{i} \neq 0, i \in\{1, \ldots, P\}$, the vector $\boldsymbol{m}_{2 P}^{*}(\sigma)$ will then have the following specific properties:

1. The vector $\boldsymbol{m}_{2 P-1}^{*}(\sigma)$ must be strictly within the realisable moment space $\mathcal{M}_{N-1}\left(\Omega_{\xi}\right)$.

2. The vector $\boldsymbol{m}_{2 P}^{*}(\sigma)$ must be on the boundary of the realisable moment space $\mathcal{M}_{N}\left(\Omega_{\xi}\right)$. EQMOM procedure will then rely on the realisability of the vector $\boldsymbol{m}_{2 P}^{*}(\sigma)$ instead of the computation of the error on the last moment, this will be a cheaper approach. The actual definition of the realisable moment space of order $n, \mathcal{M}_{n}$, depends on the support $\Omega_{\xi}$ of the NDF. The three classical supports, corresponding to the Hamburger, Stieltjes and Hausdorff moment problems, come with different constraints on a moment set to ensure its realisability. The realisability criteria for each of these supports will then be detailed.

Fig. 1 sums up the "standard approach" based on $D_{N}(\sigma)$, the shifted approach, based on $D_{N}^{*}(\sigma)$, as well as the new approach based on the realisability criteria of $\boldsymbol{m}_{2 P}^{*}(\sigma)$ for all three supports. 

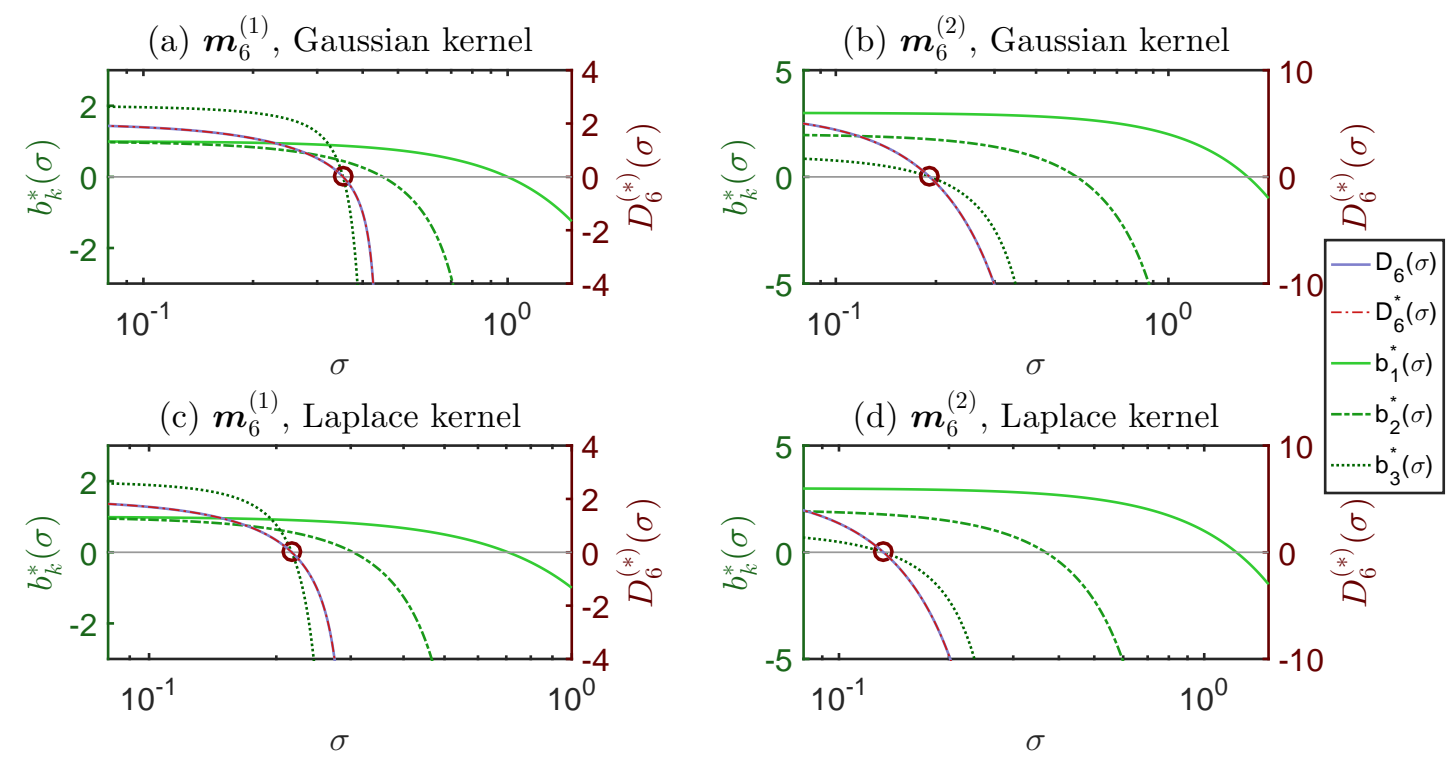

Figure 2: Evolution of the different convergence criteria for both Gaussian (a and b) and Laplace (c and d) kernels depending on $\sigma$ value. The two initial moment sets are $\boldsymbol{m}_{6}^{(1)}=\left[\begin{array}{llllllll}1 & 1 & 2 & 5 & 12 & 42 & 133\end{array}\right]^{\top}$ and $\boldsymbol{m}_{6}^{(2)}=\left[\begin{array}{lllllll}1 & 2 & 7 & 17 & 58 & 149 & 493\end{array}\right]^{\top}$.

\subsection{Application to the Hamburger problem}

As stated in 2.2, it is known that the monic polynomials which are orthogonal to a measure $\mathrm{d} \mu(\xi)=n(\xi) \mathrm{d} \xi$ satisfy a three-term recurrence relation (Eq. (5)) with $a_{k}$ and $b_{k}, k \in \mathbb{N}$, the recurrence coefficients specific to the measure $\mathrm{d} \mu(\xi)$. The Favard's theorem [28] and its converse [29] imply that the measure $\mathrm{d} \mu(\xi)$ is realisable on $\left.\Omega_{\xi}=\right]-\infty,+\infty$ [ if and only if $a_{k} \in \mathbb{R}$ and $b_{k}>0, \forall k \in \mathbb{N}$.

One looks for a value of $\sigma$ such that the associated degenerated moments $\boldsymbol{m}_{2 P-1}^{*}(\sigma)$ are within the realisable moment space and the moments $\boldsymbol{m}_{2 P}^{*}(\sigma)$ are on the boundary of this moment space. Then, if the Chebyshev algorithm is used to compute the recurrence coefficients $\boldsymbol{a}_{P-1}^{*}(\sigma)=\left[a_{0}^{*}(\sigma), \ldots, a_{P-1}^{*}(\sigma)\right]^{\top}$ and $\boldsymbol{b}_{P}^{*}(\sigma)=\left[b_{1}^{*}(\sigma), \ldots, b_{P}^{*}(\sigma)\right]^{\top}$ from the vector $\boldsymbol{m}_{2 P}^{*}(\sigma)$, the condition of realisability can be written in terms of values of $\boldsymbol{b}_{P}^{*}(\sigma)$ : looking for the EQMOM reconstruction parameters with the Gaussian and Laplace kernels is equivalent to looking for a value of $\sigma$ such as:

- $b_{k}^{*}(\sigma)>0, \quad \forall k \in\{1, \ldots, P-1\}$

- $b_{P}^{*}(\sigma)=0$

Fig. 2 makes use of the developments from Appendix B.1 and Appendix B.2, about the Gaussian and Laplace kernels respectively, to show the evolution of $D_{6}(\sigma), D_{6}^{*}(\sigma)$ and $b_{k}^{*}(\sigma), k \in\{1,2,3\}$ for two sets of 7 moments $(P=3)$. This figure illustrates the fact that indeed the approaches based on $D_{N}(\sigma), D_{N}^{*}(\sigma)$ and $b_{P}^{*}(\sigma)$ are equivalent as they share the same circled root. 
Let denote $\sigma_{k}$ the root of $b_{k}(\sigma)$. One can notice that the root $\sigma_{k}$ lies within the interval $\left[0, \sigma_{k-1}\right]$. We actually observed the existence of all roots $\sigma_{k}, k \in\{1, \ldots, P\}$ on numerous (about $10^{6}$ ) randomly selected moment sets of $N+1=13$ moments, and never observed an undefined root. The generality of this observation has not been mathematically proved, but it seems that indeed $\sigma_{k}$ is always defined and always lies in $\sigma_{k} \in\left[0, \sigma_{k-1}\right], k \in\{2, \ldots, P\}$. $\sigma_{1}$ is defined analytically.

The previous observations were used to design a simple algorithm which allows identifying the root $\sigma_{P}$. This algorithm is based on the fact that it is possible to check whether a value $\sigma_{t}$ is higher or lower than $\sigma_{P}$ at low cost and with no prior knowledge of $\sigma_{P}$ value:

- If $b_{k}^{*}\left(\sigma_{t}\right)>0, \forall k \in\{1, \ldots, P\}$, then $\sigma_{t}<\sigma_{P}$.

- Otherwise, that is if $\exists k \in\{1, \ldots, P\}, b_{k}^{*}\left(\sigma_{t}\right)<0$, then $\sigma_{t}>\sigma_{P}$.

One can then use an iterative approach that will

1. Check the realisability of the raw moments $\boldsymbol{m}_{2 P}=\boldsymbol{m}_{2 P}^{*}(0)$ by computing $\boldsymbol{b}_{P}^{*}(0)$ and checking the positivity of all elements.

2. Initialise an interval $\left[\sigma_{l}^{(0)}, \sigma_{r}^{(0)}\right]$ such that $\sigma_{l}^{(0)}<\sigma_{P}$ and $\sigma_{r}^{(0)}>\sigma_{P}$, and then update these bounds to shrink the search interval. These initial values will be $\sigma_{l}^{(0)}=0$ and $\sigma_{r}^{(0)}=\sigma_{1}$ with $\sigma_{1}$ the analytical solution of $b_{1}^{*}(\sigma)=0$.

3. Iterate over $k$

(a) Choose $\sigma_{t} \in\left[\sigma_{l}^{(k-1)}, \sigma_{r}^{(k-1)}\right]$.

(b) Compute $\boldsymbol{b}_{P}^{*}\left(\sigma_{t}\right)$.

(c) If all elements of $\boldsymbol{b}_{P}^{*}\left(\sigma_{t}\right)$ are positive, set $\sigma_{l}^{(k)}=\sigma_{t}$ and $\sigma_{r}^{(k)}=\sigma_{r}^{(k-1)}$.

(d) Otherwise, set $\sigma_{l}^{(k)}=\sigma_{l}^{(k-1)}$ and $\sigma_{r}^{(k)}=\sigma_{t}$.

The choice of $\sigma_{t}$ at step 3a will be made by trying to locate the root $\sigma_{j}$ of $b_{j}^{*}(\sigma)$ with $j$ the index of the first negative element of $\boldsymbol{b}_{P}^{*}\left(\sigma_{r}^{(k)}\right)$. Following Nguyen et al. [1] developments, the use of Ridder's method is advised to select $\sigma_{t}$. This method actually tests two $\sigma$ values per iteration. Consequently, the step 3 of the previous algorithm becomes:

3. Iterate over $k$

(a) Identify $j$ the index of the first negative element of $\boldsymbol{b}_{P}^{*}\left(\sigma_{r}^{(k-1)}\right)$.

(b) Compute $\sigma_{t_{1}}=\frac{1}{2}\left(\sigma_{l}^{(k-1)}+\sigma_{r}^{(k-1)}\right)$ and $\boldsymbol{b}_{P}^{*}\left(\sigma_{t_{1}}\right)$.

(c) Compute $\sigma_{t_{2}}=\sigma_{t_{1}}+\left(\sigma_{t_{1}}-\sigma_{l}^{(k-1)}\right) \frac{b_{j}^{*}\left(\sigma_{t_{1}}\right)}{\sqrt{b_{j}^{*}\left(\sigma_{t_{1}}\right)^{2}-b_{j}^{*}\left(\sigma_{l}^{(k-1)}\right) * b_{j}^{*}\left(\sigma_{r}^{(k-1)}\right)}}$ and $\boldsymbol{b}_{P}^{*}\left(\sigma_{t_{2}}\right)$.

(d) Set $\sigma_{l}^{(k)}$ as the highest value between $\sigma_{l}^{(k-1)}, \sigma_{t_{1}}$ and $\sigma_{t_{2}}$ such that the corresponding vector $\boldsymbol{b}_{P}^{*}$ contains only positive values.

(e) Set $\sigma_{r}^{(k)}$ as the lowest value between $\sigma_{r}^{(k-1)}, \sigma_{t_{1}}$ and $\sigma_{t_{2}}$ such that the corresponding vector $\boldsymbol{b}_{P}^{*}$ contains at least one negative value. 
Stop the computation if $\sigma_{r}^{(k)}-\sigma_{l}^{(k)}<\varepsilon \sigma_{1}$ or if $b_{P}^{*}\left(\sigma_{l}^{(k)}\right)<\varepsilon b_{P}^{*}(0)$, with $\varepsilon$ a relative tolerance (e.g. $\varepsilon=10^{-10}$ ). Then compute the weights $\boldsymbol{w}_{P}$ and nodes $\boldsymbol{\xi}_{P}$ of the EQMOM reconstruction by computing a Gauss quadrature based on the recurrence coefficients $\boldsymbol{a}_{P-1}^{*}\left(\sigma_{l}^{(k)}\right)$ and $\boldsymbol{b}_{P-1}^{*}\left(\sigma_{l}^{(k)}\right)$.

Actual implementations of this algorithm for both kernels are provided as supplementary data.

\subsection{Application to the Stieltjes problem}

It is well known that the realisability of a moment set $\boldsymbol{m}_{N}$ on the support $\left.\Omega_{\xi}=\right] 0,+\infty[$ is strictly equivalent to the positivity of the Hankel determinants $\underline{\mathcal{H}}_{2 n+d}[30]$ defined as:

$$
\underline{\mathcal{H}}_{2 n+d}=\left|\begin{array}{ccc}
m_{d} & \cdots & m_{n+d} \\
\vdots & \ddots & \vdots \\
m_{n+d} & \cdots & m_{2 n+d}
\end{array}\right|
$$

with $d \in\{0,1\}$ and $n \in \mathbb{N}, 2 n+d \leq N$.

This condition on the positivity of Hankel determinants can be translated into a condition on the positivity of the numbers $\zeta_{k}[29]$ defined by :

$$
\zeta_{k}=\frac{\underline{\mathcal{H}}_{k-3} \underline{\mathcal{H}}_{k}}{\underline{\mathcal{H}}_{k-2} \underline{\mathcal{H}}_{k-1}}, \quad \underline{\mathcal{H}}_{j}=1 \text { if } j<0
$$

These numbers can be directly computed from the recurrence coefficients $\boldsymbol{a}_{P}$ and $\boldsymbol{b}_{P}$ defined in 2.2 through the following relations:

$$
\zeta_{2 k}=\frac{b_{k}}{\zeta_{2 k-1}}, \quad \zeta_{2 k+1}=a_{k}-\zeta_{2 k}
$$

with $\zeta_{1}=a_{0}=m_{1} / m_{0}$.

The goal here is to use these realisability criteria to compute the parameters of EQMOM quadrature with either the Log-normal, the Gamma or the Weibull kernel (see Appendix B.3, Appendix B.4 and Appendix B.5 respectively). In these cases, one must

1. Compute $\boldsymbol{m}_{N}^{*}(\sigma)=\boldsymbol{A}_{N}^{-1}(\sigma) \cdot \boldsymbol{m}_{N}$ with $\boldsymbol{A}_{N}(\sigma)$ the matrix associated to the chosen kernel (see Appendix B.3, Appendix B.4, Appendix B.5).

2. Apply the Chebyshev algorithm to $\boldsymbol{m}_{N}^{*}(\sigma)$ to access the recurrence coefficients $\boldsymbol{a}_{P}^{*}(\sigma)$ and $\boldsymbol{b}_{P}^{*}(\sigma)$.

3. Compute $\boldsymbol{\zeta}_{N}^{*}(\sigma)=\left[\zeta_{1}^{*}(\sigma), \ldots, \zeta_{N}^{*}(\sigma)\right]^{\top}$ using the relations in Eq. (21).

One actually looks for $\sigma$ such that

- $\zeta_{k}^{*}(\sigma)>0, \quad \forall k \in\{1, \ldots, N-1\}$

- $\zeta_{N}^{*}(\sigma)=0$ 


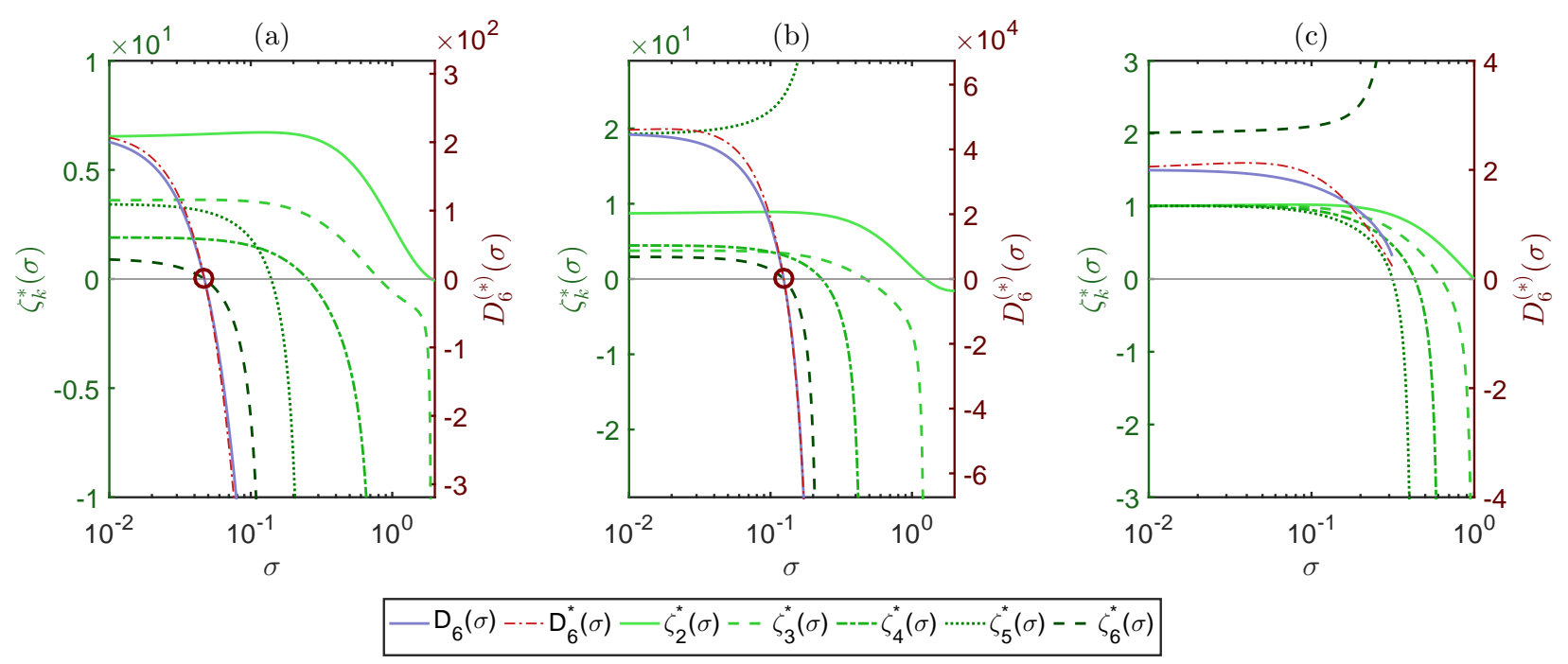

Figure 3: Evolution of the different convergence criteria for the Weibull kernel depending on $\sigma$ value. The ini-

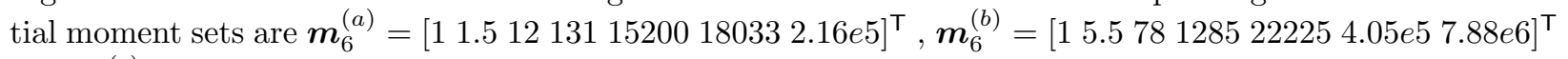
and $\boldsymbol{m}_{6}^{(c)}=\left[\begin{array}{lllllll}1 & 1 & 2 & 5 & 14 & 42 & 133\end{array}\right]^{\top}$.

Let $\sigma_{k}$ be the root of $\zeta_{k}^{*}(\sigma)$. In all cases, the root $\sigma_{2}$ is defined, analytically for the Log-normal and Gamma kernels, and numerically for the Weibull kernel. Fig. 3 shows the evolution of $D_{6}(\sigma), D_{6}^{*}(\sigma)$ and $\boldsymbol{\zeta}_{6}^{*}(\sigma)$ for three moment sets when the developments relative to the Weibull (see Appendix B.5) kernel are used. Three situations can be observed on that figure:

1. All roots $\sigma_{k}, k \in\{2, \ldots, N\}$ are defined (Fig. 3a).

2. Some intermediary roots $\sigma_{k}, k \in\{3, \ldots, N-1\}$, are not defined but the root $\sigma_{N}$ still exists (Fig. 3b).

3. The root $\sigma_{N}$ is not defined (Fig. 3c).

These three cases can be observed for the Gamma and Log-normal kernels too.

In the first two cases, when $\sigma_{N}$ exists, the EQMOM approximation is well defined. The last case -where $\zeta_{N}^{*}(\sigma)$ admits no root in $\left[0, \sigma_{N-1}\right]$ - actually corresponds to the case described by Nguyen et al. [1] where $D_{N}(\sigma)$ did not admit any root either. In this case, it was suggested to minimise $D_{N}(\sigma)$ in order to reduce the difference between $m_{N}$ and $\widetilde{m}_{N}(\sigma)$ as much as possible.

$D_{N}(\sigma)$ tends to be a decreasing function, but is undefined as soon as any element of $\boldsymbol{\zeta}_{N-1}^{*}(\sigma)$ is negative. The minimum of $D_{N}(\sigma)$ is then usually located at the highest order defined root. For instance, in the case shown in Fig. 3c, the minimum of $D_{6}(\sigma)$ is located at the root $\sigma_{5}$ of $\zeta_{5}^{*}(\sigma)$.

The moment-inversion procedure for reconstruction kernels defined on $\left.\Omega_{\xi}=\right] 0,+\infty[$ is then reduced to the identification of the defined root $\sigma_{k}, k \in\{2, \ldots, N\}$, of highest index. The algorithm proposed in section 3.3 already converges toward this root and only requires little adjustments: 
1. Check the realisability of the raw moments $\boldsymbol{m}_{2 P}=\boldsymbol{m}_{2 P}^{*}(0)$ by computing $\boldsymbol{\zeta}_{N}^{*}(0)$ and checking the positivity of all elements.

2. Initialise an interval $\left[\sigma_{l}^{(0)}, \sigma_{r}^{(0)}\right]$ with $\sigma_{l}^{(0)}=0$ and $\sigma_{r}^{(0)}=\sigma_{2}$ with $\sigma_{2}$ the solution of $\zeta_{2}^{*}(\sigma)=0$.

3. Iterate over $k$

(a) Identify $j$ the index of the first negative element of $\zeta_{N}^{*}\left(\sigma_{r}^{(k-1)}\right)$.

(b) Compute $\sigma_{t_{1}}=\frac{1}{2}\left(\sigma_{l}^{(k-1)}+\sigma_{r}^{(k-1)}\right)$ and $\boldsymbol{\zeta}_{N}^{*}\left(\sigma_{t_{1}}\right)$.

(c) Compute $\sigma_{t_{2}}=\sigma_{t_{1}}+\left(\sigma_{t_{1}}-\sigma_{l}^{(k-1)}\right) \frac{\zeta_{j}^{*}\left(\sigma_{t_{1}}\right)}{\sqrt{\zeta_{j}^{*}\left(\sigma_{t_{1}}\right)^{2}-\zeta_{j}^{*}\left(\sigma_{l}^{(k-1)}\right) * \zeta_{j}^{*}\left(\sigma_{r}^{(k-1)}\right)}}$ and $\boldsymbol{\zeta}_{N}^{*}\left(\sigma_{t_{2}}\right)$.

(d) Set $\sigma_{l}^{(k)}$ as the highest value between $\sigma_{l}^{(k-1)}, \sigma_{t_{1}}$ and $\sigma_{t_{2}}$ such that the corresponding vector $\boldsymbol{\zeta}_{N}^{*}$ contains only positive values.

(e) Set $\sigma_{r}^{(k)}$ as the lowest value between $\sigma_{r}^{(k-1)}, \sigma_{t_{1}}$ and $\sigma_{t_{2}}$ such that the corresponding vector $\boldsymbol{\zeta}_{N}^{*}$ contains at least one negative value.

Stop the computation if $\sigma_{r}^{(k)}-\sigma_{l}^{(k)}<\varepsilon \sigma_{1}$ or if $\zeta_{N}^{*}\left(\sigma_{l}^{(k)}\right)<\varepsilon \zeta_{N}^{*}(0)$, with $\varepsilon$ a relative tolerance (e.g. $\varepsilon=10^{-10}$ ). Then compute the weights $\boldsymbol{w}_{P}$ and nodes $\boldsymbol{\xi}_{P}$ of the EQMOM reconstruction by computing a Gaussian-quadrature based on the recurrence coefficients $\boldsymbol{a}_{P-1}^{*}\left(\sigma_{l}^{(k)}\right)$ and $\boldsymbol{b}_{P-1}^{*}\left(\sigma_{l}^{(k)}\right)$.

\subsection{Application to the Hausdorff problem}

The moments of a distribution defined on the closed support $\left.\Omega_{\xi}=\right] 0,1[$ must obey two sets of conditions in order to be within the realisable moment space $[12,23]$. The moment set $\boldsymbol{m}_{N}$ is interior to the realisable moment space associated to the support $\left.\Omega_{\xi}=\right] 0,1[$ if and only if:

- $\underline{\mathcal{H}}_{k}>0, \quad \forall k \in\{0, \ldots, N\}$

- $\overline{\mathcal{H}}_{k}>0, \quad \forall k \in\{1, \ldots, N\}$

with $\underline{\mathcal{H}}_{k}$ defined in Eq. (19) and $\overline{\mathcal{H}}_{k}$ defined by

$$
\overline{\mathcal{H}}_{2 n+d}=\left|\begin{array}{ccc}
m_{d-1}-m_{d} & \cdots & m_{n+d-1}-m_{n+d} \\
\vdots & \ddots & \vdots \\
m_{n+d-1}-m_{n+d} & \cdots & m_{2 n+d-1}-m_{2 n+d}
\end{array}\right|
$$

Leaving aside the obvious condition $\underline{\mathcal{H}}_{0}=m_{0}>0$, the conditions $\overline{\mathcal{H}}_{k}>0$ and $\underline{\mathcal{H}}_{k}>0$ induce a lower bound $m_{k}^{-}$and an upper bound $m_{k}^{+}$for the values of $m_{k}, k \in\{1, \ldots, N\}$. Consequently, one can define the canonical moments of the distribution $\boldsymbol{p}_{N}=\left[p_{1}, \ldots, p_{N}\right]^{\top}$ as

$$
p_{k}=\frac{m_{k}-m_{k}^{-}}{m_{k}^{+}-m_{k}^{-}}
$$


A moment set $\boldsymbol{m}_{N}$ is realisable if and only if the associated canonical moment set $\boldsymbol{p}_{N}$ lies in the hypercube $] 0,1\left[^{N}\right.$. The canonical moments can be computed through the recurrence relation [31]:

$$
p_{k}=\frac{\zeta_{k}}{1-p_{k-1}}
$$

with $\zeta_{k}$ defined in Eq. (20) and $p_{1}=m_{1}$.

In the case of the Beta kernel (see Appendix B.6), one is looking for a value of $\sigma$ such that the vector $\boldsymbol{p}_{N}^{*}(\sigma)$ has the following properties:

- $\left.p_{k}^{*}(\sigma) \in\right] 0,1[, \quad \forall k \in\{1, \ldots, N-1\}$

- $p_{N}^{*}(\sigma)=0$

$\boldsymbol{p}_{N}^{*}(\sigma)$ is computed from the vector $\boldsymbol{\zeta}_{N}^{*}(\sigma)$ which is deduced from the recurrence coefficients $\boldsymbol{a}_{P-1}^{*}(\sigma)$ and $\boldsymbol{b}_{P}^{*}(\sigma)$. These are computed -like previously- through the Chebyshev algorithm applied to the vector $\boldsymbol{m}_{N}^{*}(\sigma)=\boldsymbol{A}_{N}^{-1}(\sigma) \cdot \boldsymbol{m}_{N}$.

Fig. 4 shows the evolution of the canonical moments and the convergence criteria $D_{6}(\sigma)$ and $D_{6}^{*}(\sigma)$ for four different sets of 7 moments with the developments relative to the Beta kernel (see Appendix B.6). Each of these sets corresponds to one of the four situations encountered when dealing with Beta EQMOM:

- Fig. 4a: the root $\sigma_{N}$ of $D_{N}(\sigma), D_{N}^{*}(\sigma)$ and $p_{N}^{*}(\sigma)$ exists and can be identified through a similar procedure than that described in sections 3.3 and 3.4.

- Fig. 4b: the root $\sigma_{N}$ is not defined but the minimum of $D_{N}(\sigma)$ is located at the $\sigma$ value for which $\boldsymbol{p}_{N-1}^{*}(\sigma)$ is on the boundary of the hypercube $] 0,1\left[^{N-1}\right.$.

- Fig. 4c: $D_{N}(\sigma), D_{N}^{*}(\sigma)$ and $p_{N}^{*}(\sigma)$ admit multiple roots.

- Fig. 4d: the root $\sigma_{N}$ is defined, but there is a range $] \sigma_{v_{1}}, \sigma_{v_{2}}\left[\right.$ with $\sigma_{v_{2}}<\sigma_{N}$, highlighted in light grey, such that in this interval the convergence criteria are undefined because $\forall \sigma \in] \sigma_{v_{1}}, \sigma_{v_{2}}\left[, \boldsymbol{p}_{N-1}^{*}(\sigma) \notin\right] 0,1\left[^{N-1}\right.$.

The algorithm proposed in sections 3.3 and 3.4 can still be applied here by replacing the convergence criteria by the canonical moments, and by checking that the values of $\boldsymbol{p}_{N}^{*}(\sigma)$ all lie in the interval ]0,1[ instead of checking only for positivity:

1. Check the realisability of the raw moments $\boldsymbol{m}_{2 P}=\boldsymbol{m}_{2 P}^{*}(0)$ by computing $\boldsymbol{p}_{N}^{*}(0)$ and checking that all elements lie in $] 0,1[$.

2. Initialise an interval $\left[\sigma_{l}^{(0)}, \sigma_{r}^{(0)}\right]$ with $\sigma_{l}^{(0)}=0$ and $\sigma_{r}^{(0)}=\sigma_{2}$ with $\sigma_{2}$ the analytical solution of $p_{2}^{*}(\sigma)=0$.

3. Iterate over $k$

(a) Identify $j$ the index of the first element of $\boldsymbol{p}_{N}^{*}\left(\sigma_{r}^{(k-1)}\right)$ that is either negative or higher than 1.

(b) Compute $\sigma_{t_{1}}=\frac{1}{2}\left(\sigma_{l}^{(k-1)}+\sigma_{r}^{(k-1)}\right)$ and $\boldsymbol{p}_{N}^{*}\left(\sigma_{t_{1}}\right)$. 

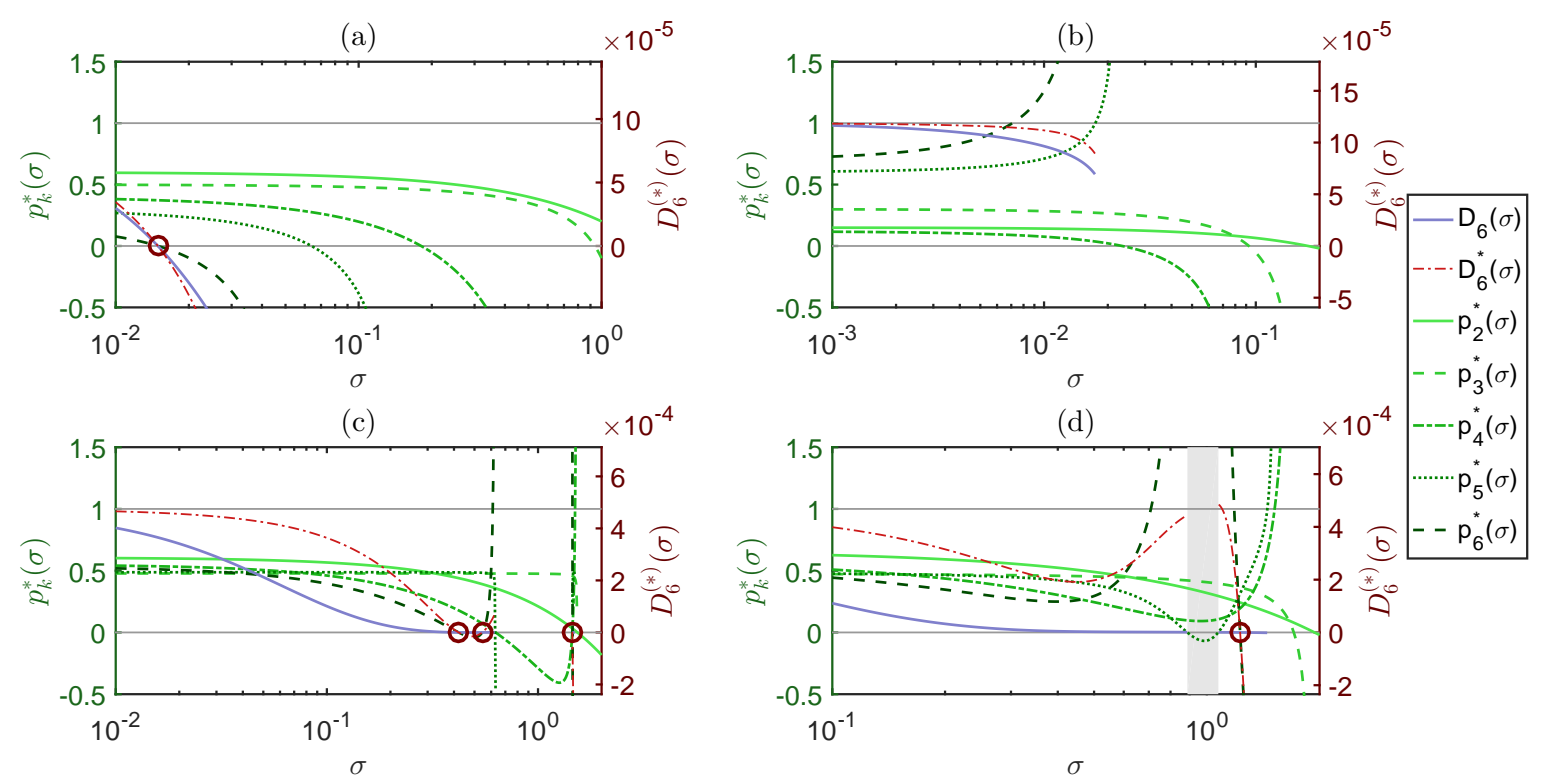

Figure 4: Evolution of the different convergence criteria for the Beta reconstruction kernel and four initial moment sets. These sets can be found in the figure source code provided as supplementary data.

(c) If $j<N$ and $p_{j}^{*}\left(\sigma_{r}^{(k-1)}\right)>1$

- Compute $\sigma_{t_{2}}=\sigma_{t_{1}}+\left(\sigma_{t_{1}}-\sigma_{l}^{(k-1)}\right) \frac{q_{j}^{*}\left(\sigma_{t_{1}}\right)}{\sqrt{q_{j}^{*}\left(\sigma_{t_{1}}\right)^{2}-q_{j}^{*}\left(\sigma_{l}^{(k-1)}\right) * q_{j}^{*}\left(\sigma_{r}^{(k-1)}\right)}}$ and $\boldsymbol{p}_{N}^{*}\left(\sigma_{t_{2}}\right)$, with $q_{j}^{*}(\sigma)=1-p_{j}^{*}(\sigma)$.

(d) Else, that is if $j=N$ or $p_{j}^{*}\left(\sigma_{r}^{(k-1)}\right)<0$

- Compute $\sigma_{t_{2}}=\sigma_{t_{1}}+\left(\sigma_{t_{1}}-\sigma_{l}^{(k-1)}\right) \frac{p_{j}^{*}\left(\sigma_{t_{1}}\right)}{\sqrt{p_{j}^{*}\left(\sigma_{t_{1}}\right)^{2}-p_{j}^{*}\left(\sigma_{l}^{(k-1)}\right) * p_{j}^{*}\left(\sigma_{r}^{(k-1)}\right)}}$ and $\boldsymbol{p}_{N}^{*}\left(\sigma_{t_{2}}\right)$.

(e) Set $\sigma_{l}^{(k)}$ as the highest value between $\sigma_{l}^{(k-1)}, \sigma_{t_{1}}$ and $\sigma_{t_{2}}$ such that the corresponding vector $\boldsymbol{p}_{N}^{*}$ lies in $] 0,1\left[^{N}\right.$.

(f) Set $\sigma_{r}^{(k)}$ as the lowest value between $\sigma_{r}^{(k-1)}, \sigma_{t_{1}}$ and $\sigma_{t_{2}}$ such that the corresponding vector $\boldsymbol{p}_{N}^{*}$ does not lie in $] 0,1\left[^{N}\right.$.

Stop the computation if $\sigma_{r}^{(k)}-\sigma_{l}^{(k)}<\varepsilon \sigma_{2}$ or if $p_{N}^{*}\left(\sigma_{l}^{(k)}\right)<\varepsilon p_{N}^{*}(0)$, with $\varepsilon$ a relative tolerance $\left(e . g . \varepsilon=10^{-10}\right)$. As previously, once convergence is achieved, the weights $\boldsymbol{w}_{P}$ and nodes $\boldsymbol{\xi}_{P}$ of the reconstruction can be obtained by computing a Gaussian quadrature rule based on the recurrence coefficients $\boldsymbol{a}_{P-1}^{*}\left(\sigma_{l}^{(k)}\right)$ and $\boldsymbol{b}_{P-1}^{*}\left(\sigma_{l}^{(k)}\right)$.

This algorithm will converge to the root $\sigma_{N}$ for cases similar to Fig. 4a; to the minimum of $D_{N}(\sigma)$ for cases similar to Fig. 4b; to one of the multiple roots for cases similar to Fig. 4c. In the case illustrated in Fig. 4d, the algorithm may or may not identify the existing root, depending on whether one of the intermediate tested $\sigma$ values lies in the greyed area. 
One could try to develop a more robust algorithm, that will always find the root if it is defined, even in the case shown in Fig. 4d. An other improvement would be to ensure a consistent result when multiple roots exist, for instance by converging toward the lowest root, so that a small perturbation in the raw moments will only cause a small change on the resulting $\sigma$ value. Nothing prevents the current algorithm from converging toward one root for a moment set and toward another one after a small perturbation of this set which could induce instabilities in large-scale simulations. Note that these limitations already existed in previous EQMOM implementations and do not result from the new approach developed in this article.

\section{Comparison of EQMOM approaches}

\subsection{Method}

The new EQMOM moment-inversion procedure only requires computation of the realisability criteria of the vector of degenerated moments $\boldsymbol{m}_{2 P}^{*}(\sigma)$ in order to identify $\sigma$. These computations were already performed in the original approach [1] to ensure the realisability of the vector $\boldsymbol{m}_{2 P-1}^{*}(\sigma)$ prior to the quadrature computation and ulterior steps.

It is therefore obvious that the new approach will always require a lower number of floating point operations (FLOP). In order to quantify this reduction on FLOP number, and the actual performance gain, two implementations of the Gauss EQMOM moment-inversion procedure are compared.

The first tested implementation is the one described in Fig. 1b, whose computational cost is similar to that of Nguyen et al. [1]. As it only requires the matrix $\boldsymbol{A}_{2 P}^{-1}(\sigma)$, it will benefit the same optimizations as the second approach as far as the linear system is concerned. The second tested implementation is the one described in section 3.3, based on the realisability of $\boldsymbol{m}_{2 P}^{*}(\sigma)$ through the computation of the recurrence coefficients $\boldsymbol{a}_{P-1}^{*}(\sigma)$ and $\boldsymbol{b}_{P}^{*}(\sigma)$.

Both approaches are implemented in MATLAB [19] functions which take as input a vector of moments (size $2 P+1 \times 1$ ) and returns the vectors $\boldsymbol{w}_{P}, \boldsymbol{\xi}_{P}($ size $P \times 1$ ) and the scalar $\sigma$. These implementations integrate a simple FLOP counter distinguishing each operation $(+,-, *, /, \exp , \sqrt{ })$ and counting the number of call to these operations for each step of computation (linear system, Chebyshev algorithm, quadrature computation and others).

In order to evaluate the number of operations used in the computation of the eigenvalues and eigenvectors of the Jacobi matrix (Eq. (6)), the Jacobi and the Francis algorithms which are suited for symmetric matrices [32] are used in place of the MATLAB built-in "eig" function [19]. Finally, the number of tested $\sigma$ values (i.e. the number of calls to the linear system $\left.\boldsymbol{m}_{2 P}^{*}(\sigma)=\boldsymbol{A}_{2 P}^{-1}(\sigma) \cdot \boldsymbol{m}_{2 P}\right)$ is measured too.

$10^{4}$ realisable sets of 11 moments were randomly generated through a two step process:

1. Generate two random vectors $\boldsymbol{a}_{4}$ and $\boldsymbol{b}_{5}$.

- Elements of $\boldsymbol{a}_{4}$ are distributed along a normal distribution: $a_{k} \sim \mathcal{N}(0,25), \quad k \in$ $\{0, \ldots, 4\}$.

- Elements of $\boldsymbol{b}_{5}$ are distributed along an exponential distribution $b_{k} \sim \operatorname{Exp}(5), \quad k \in$ $\{1, \ldots, 5\}$. 
2. Use a reversed Chebyshev algorithm to compute the vector of moments $\boldsymbol{m}_{10}$ corresponding to $\boldsymbol{a}_{4}$ and $\boldsymbol{b}_{5}$.

A routine applied both moment-inversion procedures on all generated moment sets and varied the actual number of moments $2 P+1 \in\{5,7,9,11\}$. This routine also measured the wall-time of each of these calls.

\subsection{Results}

Results of the comparison are given in Table 1. The Jacobi algorithm was the fastest one to compute the eigenvalues and eigenvectors in the cases $P=2$ and $P=3$ whilst the Francis algorithm was faster for $P=4$ and $P=5$. Table 1 only shows the results corresponding to that fastest algorithm for each case, in order to have the lowest estimate in FLOP and run-time gain between both implementations.

The first main observation is a decrease in the number of tested $\sigma$ values. This decrease is due to the fact that in the former approach, if $\boldsymbol{m}_{N-1}^{*}(\sigma)$ turns out not to be realisable, the objective function is set to a arbitrarily high negative value. The use of such an arbitrary value slows down the convergence of the non-linear equation solver. Meanwhile, the new approach never makes use of arbitrary values, all the elements of the vector $\boldsymbol{b}_{P}^{*}(\sigma)$ are used one after the other which yields a better choice of the next tested $\sigma$ value.

The second observation was expected and is a significant drop in the total number of FLOP. This is mainly justified by the fact that the quadrature computation is only called once in the new approach whilst it is called for most tested $\sigma$ values in the former momentinversion procedure. This quadrature, which consists in the computation of the eigenvalues and eigenvectors of a tridiagonal symmetric matrix, is the most expensive operation used in the EQMOM moment-inversion procedure.

Overall, one observes a net decrease in the number of floating-point operations and in the computation run-time of $80 \%$ to $85 \%$ for these implementations of Gauss EQMOM and the tested $10^{4}$ moment sets.

\section{Conclusion}

The first developments relative to the Extended Quadrature Method of Moments are quite recent [14]. Most of these developments were dedicated to widening the use of this method to new application cases, in particular by adding new reconstruction kernels to the EQMOM formalism, and to demonstrate its stability and accuracy compared to other methods. This article summarised all of these developments, relative to the Gaussian kernel [14], to the Log-normal kernel [18] and to the Gamma and Beta kernels [15]. It was also shown that at least two other kernels are perfectly compatible with the EQMOM formalism: the Laplace and Weibull kernels. In a previous work, the solution of a PBE in some specific setups was a Laplace-like distribution [27]. Moreover, the Weibull distribution is often met in the modelling of biological systems. We then hope that the scientific community will find a good use for these developments.

The youth of EQMOM explains that there is still room left for improvements. The core of this method -the moment-inversion procedure- is an iterative process which is its computational bottleneck. Nguyen et al. [1] proposed some modifications, compared to 
Table 1: Table of comparison of the Gauss EQMOM implementations corresponding to Fig. 1b and 1c. The count of FLOP details the operations related to (i) the matrix-vector product $\boldsymbol{A}_{2 P}^{-1}(\sigma) \cdot \boldsymbol{m}_{2 P}$, (ii) the Chebyshev Algorithm (CA), (iii) the Quadrature Computation (QC) and (iv) a miscellaneous category. Results are given as mean \pm standard-deviation.

\begin{tabular}{|c|c|c|c|c|c|c|}
\hline & & & $P=2$ & $P=3$ & $P=4$ & $P=5$ \\
\hline New & \multirow{5}{*}{ FLOP } & $\boldsymbol{A}_{2 P}^{-1}(\sigma)$ & $282_{ \pm 123}$ & $843_{ \pm 210}$ & $1842_{ \pm 328}$ & $3418_{ \pm 527}$ \\
\hline \multirow[t]{6}{*}{ approach } & & $\mathrm{CA}$ & $227_{ \pm 92}$ & $564_{ \pm 134}$ & $1146_{ \pm 195}$ & $2026_{ \pm 301}$ \\
\hline & & $\mathrm{QC}$ & $52_{ \pm 1}$ & $546_{ \pm 56}$ & $1225_{ \pm 144}$ & $2151_{ \pm 213}$ \\
\hline & & Misc. & $93_{ \pm 37}$ & $100_{ \pm 23}$ & $116_{ \pm 19}$ & $131_{ \pm 18}$ \\
\hline & & Total & $654_{ \pm 252}$ & $2053_{ \pm 357}$ & $4328_{ \pm 536}$ & $7727_{ \pm 834}$ \\
\hline & \multicolumn{2}{|c|}{ Evaluations } & $15_{ \pm 6}$ & $17_{ \pm 4}$ & $19_{ \pm 3}$ & $21_{ \pm 3}$ \\
\hline & \multicolumn{2}{|c|}{ Run-time (ms) } & $6_{ \pm 3}$ & $13_{ \pm 5}$ & $23_{ \pm 6}$ & $33_{ \pm 7}$ \\
\hline Former & \multirow{5}{*}{ FLOP } & $\boldsymbol{A}_{2 P}^{-1}(\sigma)$ & $298_{ \pm 164}$ & $1095_{ \pm 391}$ & $2683_{ \pm 741}$ & $5352_{ \pm 1450}$ \\
\hline \multirow[t]{6}{*}{ approach } & & $\mathrm{CA}$ & $223_{ \pm 115}$ & $724_{ \pm 246}$ & $1662_{ \pm 442}$ & $3184_{ \pm 836}$ \\
\hline & & $\mathrm{QC}$ & $823_{ \pm 421}$ & $9485_{ \pm 3245}$ & $23734_{ \pm 5387}$ & $46870_{ \pm 10002}$ \\
\hline & & Misc. & $228_{ \pm 119}$ & $395_{ \pm 137}$ & $634_{ \pm 155}$ & $959_{ \pm 224}$ \\
\hline & & Total & $1572_{ \pm 818}$ & $11700_{ \pm 3939}$ & $28713_{ \pm 6467}$ & $56365_{ \pm 12138}$ \\
\hline & \multicolumn{2}{|c|}{ Evaluations } & $16_{ \pm 8}$ & $21_{ \pm 7}$ & $27_{ \pm 7}$ & $32_{ \pm 9}$ \\
\hline & \multicolumn{2}{|c|}{ Run-time (ms) } & $15_{ \pm 9}$ & $73_{ \pm 30}$ & $105_{ \pm 28}$ & $162_{ \pm 40}$ \\
\hline \multirow[t]{3}{*}{ Gain in } & \multicolumn{2}{|c|}{ FLOP } & $58.4 \% \pm 26.9 \%$ & $82.5 \% \pm 6.6 . \%$ & $84.9 \% \pm 3.9 \%$ & $86.3 \% \pm 3.3 \%$ \\
\hline & \multicolumn{2}{|c|}{ Evaluations } & $6.3 \% \pm 60.0 \%$ & $19.0 \% \pm 33.0 \%$ & $29.6 \% \pm 21.4 \%$ & $34.4 \% \pm 20.7 \%$ \\
\hline & \multicolumn{2}{|c|}{ Run-time } & $60.0 \% \pm 31.2 \%$ & $82.2 \% \pm 10.0 \%$ & $78.1 \% \pm 8.2 \%$ & $79.6 \% \pm 6.6 \%$ \\
\hline
\end{tabular}

previous implementations, in order to stabilise the method and to speed-up its resolution, namely the use of Ridder's method instead of bounded-secant or dichotomic methods to solve the non-linear problem, and the realisability checks performed prior to the quadrature computation.

Further improvements were proposed by shifting the resolution toward a new paradigm. This results in a significant decrease in computational cost of about $80 \%-85 \%$ in terms of required floating-point operations. This resulted in our MATLAB implementations in a similar gain in terms of computation wall-time.

In multiple works $[1,15,27]$, EQMOM has been compared to other methods (Maximum Entropy approach or sectional methods) and exhibited (i) similar accuracy even with a lower number of resolved variables, and (ii) faster or comparable computation times. The new improvements of EQMOM will make it even more competitive as its stability and accuracy are kept while reducing the gap in terms of numerical cost between EQMOM and other cheaper methods such as Gauss or Gauss-Radau quadratures.

We strongly believe that transparency about these developments will help further refinements of EQMOM. For that reason, all sources used to generate figures and data in this article are provided as supplementary data. We also release all our EQMOM source codes both with this article and in an open-access GIT repository (url: https://gitlab.com/open-eqmom). It will be updated as well as supplemented with implementations of EQMOM in languages other than MATLAB. In the case of the Beta reconstruction kernel, some suggestions for further improvements in terms of accuracy and stability were listed in section 3.5. These 
will be tackled in ulterior work.

\section{Acknowledgments}

The authors thank Sanofi Chimie - C\&BD Biochemistry Vitry for its financial support. The authors would also like to thank Prof. Frédérique Laurent and Prof. Rodney O. Fox for the precious discussions and insights about EQMOM and the developments presented in this paper. The authors declare no conflict of interest.

\section{References}

[1] T. Nguyen, F. Laurent, R. Fox, M. Massot, Solution of population balance equations in applications with fine particles: Mathematical modeling and numerical schemes, J. Comput. Phys. 325 (2016) 129-156, ISSN 0021-9991, doi:10.1016/j.jcp.2016.08.017.

[2] P. Moilanen, M. Laakkonen, O. Visuri, V. Alopaeus, J. Aittamaa, Modelling mass transfer in an aerated $0.2 \mathrm{~m} 3$ vessel agitated by Rushton, Phasejet and Combijet impellers, Chem. Eng. J. 142 (1) (2008) 95-108, ISSN 1385-8947, doi:10.1016/j.cej.2008.01.033.

[3] A. Buffo, V. Alopaeus, A novel simplified multivariate PBE solution method for mass transfer problems, Chem. Eng. Sci. 172 (Supplement C) (2017) 463-475, ISSN 00092509, doi:10.1016/j.ces.2017.06.036.

[4] J. Morchain, J. Gabelle, A. Cockx, A coupled population balance model and CFD approach for the simulation of mixing issues in lab-scale and industrial bioreactors, AIChE J. 60 (1) (2014) 27-40, ISSN 1547-5905, doi:10.1002/aic.14238.

[5] A. Heins, R. L. Fernandes, K. V. Gernaey, A. E. Lantz, Experimental and in silico investigation of population heterogeneity in continuous Sachharomyces cerevisiae scaledown fermentation in a two-compartment setup, J. Chem. Technol. Biotechnol. 90 (2) (2015) 324-340, ISSN 1097-4660, doi:10.1002/jctb.4532.

[6] P. Fede, O. Simonin, P. Villedieu, Monte-Carlo simulation of colliding particles or coalescing droplets transported by a turbulent flow in the framework of a joint fluidparticle pdf approach, Int. J. Multiph. Flow 74 (Supplement C) (2015) 165-183, ISSN 0301-9322, doi:10.1016/j.ijmultiphaseflow.2015.04.006.

[7] S. Kumar, D. Ramkrishna, On the solution of population balance equations by discretization - I. A fixed pivot technique, Chem. Eng. Sci. 51 (8) (1996) 1311 - 1332 , ISSN 0009-2509, doi:10.1016/0009-2509(96)88489-2.

[8] S. Kumar, D. Ramkrishna, On the solution of population balance equations by discretization - II. A moving pivot technique, Chem. Eng. Sci. 51 (8) (1996) 1333-1342, ISSN 0009-2509, doi:10.1016/0009-2509(95)00355-X.

[9] M. Massot, F. Laurent, D. K. S. de Chaisemartin, A robust moment method for evaluation of the disappearance rate of evaporating sprays, SIAM J. Appl. Math. 70 (8) (2010) 3203-3234, doi:10.1137/080740027. 
[10] V. John, I. Angelov, A. Öncül, D. Thévenin, Techniques for the reconstruction of a distribution from a finite number of its moments, Chem. Eng. Sci. 62 (11) (2007) 28902904, ISSN 0009-2509, doi:10.1016/j.ces.2007.02.041.

[11] L. Mead, N. Papanicolaou, Maximum entropy in the problem of moments, J. Math. Phys. 25 (8) (1984) 2404-2417.

[12] A. Tagliani, Hausdorff moment problem and maximum entropy: A unified approach, Appl. Math. Comput. 105 (2-3) (1999) 291-305, ISSN 0096-3003, doi:10.1016/ S0096-3003(98)10084-X.

[13] G. Athanassoulis, P. Gavriliadis, The truncated Hausdorff moment problem solved by using kernel density functions, Probab. Eng. Mech. 17 (3) (2002) 273-291, ISSN 02668920, doi:10.1016/S0266-8920(02)00012-7.

[14] C. Chalons, R. Fox, M. Massot, A multi-Gaussian quadrature method of moments for gas-particle flows in a LES framework, in: Proceedings of the 2010 Summer Program, Center for turbulence Research, Stanford University, 347-358, 2010.

[15] C. Yuan, F. Laurent, R. Fox, An extended quadrature method of moments for population balance equations, J. Aerosol Sci. 51 (0) (2012) 1-23, ISSN 0021-8502, doi: 10.1016/j.jaerosci.2012.04.003.

[16] A. Passalacqua, F. Laurent, E. Madadi-Kandjani, J. Heylmun, R. Fox, An open-source quadrature-based population balance solver for OpenFOAM, Chem. Eng. Sci. ISSN 0009-2509, doi:10.1016/j.ces.2017.10.043.

[17] D. Marchisio, R. Fox, Computational Models for Polydisperse Particulate and Multiphase Systems, Cambridge Series in Chemical Engineering, Cambridge University Press, ISBN 9781107328174, 2013.

[18] E. Madadi-Kandjani, A. Passalacqua, An extended quadrature-based moment method with log-normal kernel density functions, Chem. Eng. Sci. 131 (0) (2015) 323-339, ISSN 0009-2509, doi:10.1016/j.ces.2015.04.005.

[19] MATLAB, version 9.0 (R2016a), The MathWorks, Inc., Natick, Massachusetts, United States, 2016.

[20] R. McGraw, Description of Aerosol Dynamics by the Quadrature Method of Moments, Aerosol Sci. Technol. 27 (2) (1997) 255-265, doi:10.1080/02786829708965471.

[21] W. Gautschi, Orthogonal Polynomials: Computation and Approximation, Numerical mathematics and scientific computation, Oxford University Press, ISBN 9780198506720, 2004.

[22] P. Henrici, The Quotient-Difference algorithm, Natl. Bur. Stand., Appl. Math. Ser. 49 (1958) 23-46. 
[23] H. Dette, W. Studden, The theory of canonical moments with applications in statistics, probability, and analysis, John Wiley \& Sons, New York; Chichester, 1997.

[24] R. Gordon, Error Bounds in Equilibrium Statistical Mechanics, J. Math. Phys. 9 (1968) 655, doi:10.1063/1.1664624.

[25] J. Wheeler, Modified moments and Gaussian quadratures, Rocky Mountain J. Math. 4 (2) (1974) 287-296, doi:RMJ-1974-4-2-287.

[26] N. Lebaz, A. Cockx, M. Spérandio, J. Morchain, Reconstruction of a distribution from a finite number of its moments: A comparative study in the case of depolymerization process, Comput. Chem. Eng. 84 (2016) 326-337, ISSN 0098-1354, doi: 10.1016/j.compchemeng.2015.09.008.

[27] M. Pigou, J. Morchain, P. Fede, M. Penet, G. Laronze, An assessment of methods of moments for the simulation of population dynamics in large-scale bioreactors, Chem. Eng. Sci. 171 (2017) 218-232, ISSN 0009-2509, doi:10.1016/j.ces.2017.05.026.

[28] J. Favard, Sur les polynomes de Tchebicheff, C. r. hebd. séances Acad. sci. 200 (1935) 2052-2053.

[29] T. Chihara, An introduction to orthogonal polynomials, no. vol. 13 in Mathematics and its applications, Gordon and Breach, ISBN 9780677041506, 1978.

[30] J. Shohat, J. Tamarkin, The Problem of Moments, Mathematical Surveys and Monographs, American Mathematical Society, 4 edn., 1943.

[31] H. Wall, Analytic Theory of Continued Fractions, The University series in higher mathematics, Van Nostrand, 1948.

[32] W. Ford, Chapter 19 - The Symmetric Eigenvalue Problem, in: Numerical Linear Algebra with Applications, Academic Press, Boston, ISBN 978-0-12-394435-1, 439-468, doi:10.1016/B978-0-12-394435-1.00019-3, 2015.

[33] M. Wilck, A general approximation method for solving integrals containing a lognormal weighting function, J. Aerosol Sci. 32 (9) (2001) 1111-1116, ISSN 0021-8502, doi:10. 1016/S0021-8502(01)00044-1.

[34] J. Shen, T. Tang, L.-L. Wang, Orthogonal Polynomials and Related Approximation Results, Springer Berlin Heidelberg, Berlin, Heidelberg, ISBN 978-3-540-71041-7, 47140, doi:10.1007/978-3-540-71041-7_3, 2011. 


\section{Appendix A. Chebyshev algorithm}

The Chebyshev algorithm allows to compute the three-term recurrence coefficients of the monic polynomials orthogonal to a measure $\mathrm{d} \mu(\xi)$ whose moments are given by the vector $\boldsymbol{m}_{N}=\left[m_{0}, \ldots, m_{N}\right]$. This version of the algorithm fills column-wise a $N+1 \times\left\lceil\frac{N+1}{2}\right\rceil$ matrix denoted $\boldsymbol{S}$.

First, fill the first column with the moments $S_{i, 0}=m_{i}$, compute $a_{0}=m_{1} / m_{0}$ and fill the second column with $S_{i, 1}=S_{i+1,0}-a_{0} S_{i, 0}, \quad \forall i \in\{1, \ldots, N-1\}$.

Then iterate for $j \in\left\{2, \ldots,\left\lceil\frac{N-1}{2}\right\rceil\right\}$ :

$$
\begin{aligned}
a_{j-1} & =\frac{S_{j, j-1}}{S_{j-1, j-1}}-\frac{S_{j-1, j-2}}{S_{j-2, j-2}} \\
b_{j-1} & =\frac{S_{j-1, j-1}}{S_{j-2, j-2}} \\
S_{i, j} & =S_{i+1, j-1}-a_{j-1} S_{i, j-1}-b_{j-1} S_{i, j-2}, \quad i \in\{j, \ldots, N-j\}
\end{aligned}
$$

\section{Appendix B. Kernels for EQMOM}

There exists multiple variations of the EQMOM method depending on the Kernel Density Function that is used for the reconstruction in Eq. (15). This section details the specificities of multiple KDF that were found to be compatible with the EQMOM procedure. It details for each kernel

1. the actual expression of that kernel $\delta_{\sigma}\left(\xi, \xi_{m}\right)$;

2. the expression of its moments;

3. the matrix $\boldsymbol{A}_{n}(\sigma)$ that allows the transfer between the raw moments of the reconstruction $\widetilde{\boldsymbol{m}}_{n}$ and its degenerated moments $\boldsymbol{m}_{n}^{*}$;

4. the nested quadrature rules suiting this kernel;

5. the analytical solutions available for one-node EQMOM $(P=1)$.

Two-nodes analytical solutions exist for the Gaussian, Gamma, Laplace and Log-normal kernels and are accessible using the same methodology than that used by Chalons et al. [14] for the Gaussian kernel. These solutions are not detailed here but are implemented in the MATLAB code given in supplementary data.

All definitions of matrices $A_{n}(\sigma)$ are given using zero-offset. The element of the first line and column of this matrix then reads $A_{0,0}(\sigma)$.

\section{Appendix B.1. Gaussian kernel}

Appendix B.1.1. Definition

The Gaussian kernel $\delta_{\sigma}^{(G)}\left(\xi, \xi_{m}\right)$ was first used in EQMOM by Chalons et al. [14]. It is defined on $\left.\Omega_{\xi}=\right]-\infty,+\infty[$ by

$$
\delta_{\sigma}^{(G)}\left(\xi, \xi_{m}\right)=\frac{1}{\sigma \sqrt{2 \pi}} \exp \left(-\frac{\left(\xi-\xi_{m}\right)^{2}}{2 \sigma^{2}}\right)
$$


Appendix B.1.2. Moments and linear system

Moments of the Gaussian kernel are given by:

$$
\int_{-\infty}^{+\infty} \xi^{k} \delta_{\sigma}^{(G)}\left(\xi, \xi_{m}\right) \mathrm{d} \xi=\sum_{j=0}^{\lfloor k / 2\rfloor} \frac{k !}{j !(k-2 j) !}\left(\frac{\sigma^{2}}{2}\right)^{j} \xi_{m}^{k-2 j}
$$

Moments of the distribution $\widetilde{n}(\xi)=\sum_{i=1}^{P} w_{i} \delta_{\sigma}^{(G)}\left(\xi, \xi_{i}\right)$ are given by the linear system

$$
\widetilde{\boldsymbol{m}}_{n}=\boldsymbol{A}_{n}^{(G)}(\sigma) \cdot \boldsymbol{m}_{n}^{*}
$$

with

$$
A_{i, j}^{(G)}(\sigma)= \begin{cases}0 & \text { if } j>i \text { or }(i-j \quad \bmod 2)=1 \\ \frac{i !}{\left(\frac{i-j}{2}\right) ! j !}\left(\frac{\sigma^{2}}{2}\right)^{\frac{i-j}{2}} & \text { otherwise }\end{cases}
$$

The inverse of this matrix is given by:

$$
A_{i, j}^{(G)-1}(\sigma)= \begin{cases}0 & \text { if } j>i \text { or }(i-j \quad \bmod 2)=1 \\ \frac{i !}{\left(\frac{i-j}{2}\right) ! j !}\left(-\frac{\sigma^{2}}{2}\right)^{\frac{i-j}{2}} & \text { otherwise }\end{cases}
$$

which translates, for the case $n=4$ into:

$$
\begin{aligned}
& {\left[\begin{array}{l}
\widetilde{m}_{0} \\
\widetilde{m}_{1} \\
\widetilde{m}_{2} \\
\widetilde{m}_{3} \\
\widetilde{m}_{4}
\end{array}\right]=\left(\begin{array}{ccccc}
1 & & & & 0 \\
0 & 1 & & & \\
\sigma^{2} & 0 & 1 & & \\
0 & 3 \sigma^{2} & 0 & 1 & \\
3 \sigma^{4} & 0 & 6 \sigma^{2} & 0 & 1
\end{array}\right) \cdot\left[\begin{array}{l}
m_{0}^{*} \\
m_{1}^{*} \\
m_{2}^{*} \\
m_{3}^{*} \\
m_{4}^{*}
\end{array}\right]} \\
& {\left[\begin{array}{c}
m_{0}^{*} \\
m_{1}^{*} \\
m_{2}^{*} \\
m_{3}^{*} \\
m_{4}^{*}
\end{array}\right]=\left(\begin{array}{ccccc}
1 & & & & 0 \\
0 & 1 & & & \\
-\sigma^{2} & 0 & 1 & & \\
0 & -3 \sigma^{2} & 0 & 1 & \\
3 \sigma^{4} & 0 & -6 \sigma^{2} & 0 & 1
\end{array}\right) \cdot\left[\begin{array}{l}
\widetilde{m}_{0} \\
\widetilde{m}_{1} \\
\widetilde{m}_{2} \\
\widetilde{m}_{3} \\
\widetilde{m}_{4}
\end{array}\right]}
\end{aligned}
$$

Appendix B.1.3. Moment preserving nested quadrature

The approximation of integral properties using Gauss EQMOM is performed through the following nested quadrature:

$$
\int_{-\infty}^{+\infty} f(\xi) n(\xi) \mathrm{d} \xi \approx \frac{1}{\sqrt{\pi}} \sum_{i=1}^{P} w_{i} \sum_{j=1}^{Q} \omega_{j} f\left(\xi_{i}+\sigma \lambda_{j} \sqrt{2}\right)
$$

with $\boldsymbol{w}_{P}, \boldsymbol{\xi}_{P}$ and $\sigma$ the EQMOM reconstruction parameters computed from $\boldsymbol{m}_{2 P} ; \boldsymbol{\omega}_{Q}$ and $\boldsymbol{\lambda}_{Q}$ are the weights and nodes of a $Q$-nodes Gauss-Hermite quadrature rule (see Appendix $\mathrm{C})$. 
Appendix B.1.4. Single node analytical solution

The case $P=1$ has the following analytical solution:

$$
\begin{aligned}
w_{1} & =m_{0} \\
\xi_{1} & =\frac{m_{1}}{m_{0}} \\
\sigma & =\frac{\sqrt{m_{2} m_{0}-m_{1}^{2}}}{m_{0}}
\end{aligned}
$$

Appendix B.2. Laplace kernel

Appendix B.2.1. Definition

The Laplace kernel $\delta_{\sigma}^{(\lambda)}\left(\xi, \xi_{m}\right)$ is defined on $\left.\Omega_{\xi}=\right]-\infty,+\infty[$ by

$$
\delta_{\sigma}^{(\lambda)}\left(\xi, \xi_{m}\right)=\frac{1}{2 \sigma} \exp \left(-\frac{\left|\xi-\xi_{m}\right|}{\sigma}\right)
$$

Appendix B.2.2. Moments and linear system

Moments of the Laplace kernel are given by

$$
\int_{-\infty}^{+\infty} \xi^{k} \delta_{\sigma}^{(\lambda)}\left(\xi, \xi_{m}\right) \mathrm{d} \xi=\sum_{j=0}^{k} \frac{k !}{(k-j) !} \frac{1+(-1)^{j}}{2} \xi_{m}^{k-j} \sigma^{j}
$$

77 Moments of the distribution $\widetilde{n}(\xi)=\sum_{i=1}^{P} w_{i} \delta_{\sigma}^{(\lambda)}\left(\xi, \xi_{i}\right)$ are given by the linear system

$$
\widetilde{\boldsymbol{m}}_{n}=\boldsymbol{A}_{n}^{(\lambda)}(\sigma) \cdot \boldsymbol{m}_{n}^{*}
$$

with

$$
A_{i, j}^{(\lambda)}(\sigma)= \begin{cases}0 & \text { if } j>i \text { or }(i-j \quad \bmod 2)=1 \\ \frac{i !}{j !} \sigma^{i-j} & \text { otherwise }\end{cases}
$$

The inverse matrix is defined by

$$
A_{i, j}^{(\lambda)}{ }^{-1}(\sigma)= \begin{cases}1 & \text { if } i=j \\ -(j+1)(j+2) \sigma^{2} & \text { if } i=j+2 \\ 0 & \text { otherwise }\end{cases}
$$

which translates for $n=6$ into 


$$
\begin{aligned}
& \left(\begin{array}{c}
\widetilde{m}_{0} \\
\widetilde{m}_{1} \\
\widetilde{m}_{2} \\
\widetilde{m}_{3} \\
\widetilde{m}_{4} \\
\widetilde{m}_{5} \\
\widetilde{m}_{6}
\end{array}\right)=\left(\begin{array}{ccccccc}
1 & & & & & & 0 \\
0 & 1 & & & & & \\
\frac{2 ! \sigma^{2}}{0 !} & 0 & 1 & & & & \\
0 & \frac{3 ! \sigma^{2}}{1 !} & 0 & 1 & & & \\
\frac{4 ! \sigma^{4}}{0 !} & 0 & \frac{4 ! \sigma^{2}}{2 !} & 0 & 1 & & \\
0 & \frac{5 ! \sigma^{4}}{1 !} & 0 & \frac{5 ! \sigma^{2}}{3 !} & 0 & 1 & \\
\frac{6 ! \sigma^{6}}{0 !} & 0 & \frac{6 ! \sigma^{4}}{2 !} & 0 & \frac{6 ! \sigma^{2}}{4 !} & 0 & 1
\end{array}\right) \quad \cdot\left(\begin{array}{c}
m_{0}^{*} \\
m_{1}^{*} \\
m_{2}^{*} \\
m_{3}^{*} \\
m_{4}^{*} \\
m_{5}^{*} \\
m_{6}^{*}
\end{array}\right) \\
& \left(\begin{array}{c}
m_{0}^{*} \\
m_{1}^{*} \\
m_{2}^{*} \\
m_{3}^{*} \\
m_{4}^{*} \\
m_{5}^{*} \\
m_{6}^{*}
\end{array}\right)=\left(\begin{array}{ccccccc}
1 & & & & & & 0 \\
0 & 1 & & & & \\
-2 \sigma^{2} & 0 & 1 & & & \\
& -6 \sigma^{2} & 0 & 1 & & \\
& & -12 \sigma^{2} & 0 & 1 & & \\
0 & & & -20 \sigma^{2} & 0 & 1 & \\
0 & & & & -30 \sigma^{2} & 0 & 1
\end{array}\right) \cdot \cdot\left(\begin{array}{c}
\widetilde{m}_{0} \\
\widetilde{m}_{1} \\
\widetilde{m}_{2} \\
\widetilde{m}_{3} \\
\widetilde{m}_{4} \\
\widetilde{m}_{5} \\
\widetilde{m}_{6}
\end{array}\right)
\end{aligned}
$$

Appendix B.2.3. Moment preserving nested quadrature

The approximation of integral properties using Laplace EQMOM is performed through the following nested quadrature:

$$
\int_{-\infty}^{+\infty} f(\xi) n(\xi) \mathrm{d} \xi \approx \sum_{i=1}^{P} w_{i} \sum_{j=1}^{Q} \omega_{j} f\left(\xi_{i}+\sigma \lambda_{j}\right)
$$

with $\boldsymbol{w}_{P}, \boldsymbol{\xi}_{P}$ and $\sigma$ the EQMOM reconstruction parameters computed from $\boldsymbol{m}_{2 P} ; \boldsymbol{\omega}_{Q}$ and $\boldsymbol{\lambda}_{Q}$ are the weights and nodes of a $Q$-nodes "Gauss-Laplace" quadrature rule (see Appendix $\mathrm{C})$.

Appendix B.2.4. Single node analytical solution

The case $P=1$ has the following analytical solution:

$$
\begin{aligned}
w_{1} & =m_{0} \\
\xi_{1} & =\frac{m_{1}}{m_{0}} \\
\sigma & =\sqrt{\frac{m_{2} m_{0}-m_{1}^{2}}{2 m_{0}^{2}}}
\end{aligned}
$$

Appendix B.3. Log-normal kernel

Appendix B.3.1. Definition

The Log-normal kernel $\delta_{\sigma}^{(L)}\left(\xi, \xi_{m}\right)$ was first used in EQMOM by Madadi-Kandjani and Passalacqua [18]. It is defined on $\left.\Omega_{\xi}=\right] 0,+\infty[$ by 


$$
\delta_{\sigma}^{(L)}\left(\xi, \xi_{m}\right)=\frac{1}{\sigma \xi \sqrt{2 \pi}} \exp \left(-\frac{\left(\log (\xi)-\log \left(\xi_{m}\right)\right)^{2}}{2 \sigma^{2}}\right)
$$

\section{Appendix B.3.2. Moments and linear system}

Moments of the Log-normal kernel are given by

$$
\int_{0}^{+\infty} \xi^{k} \delta_{\sigma}^{(L)}\left(\xi, \xi_{m}\right) \mathrm{d} \xi=\xi_{m}^{k} z^{k^{2}} \quad \text { with } z=e^{\sigma^{2} / 2}
$$

Moments of the distribution $\widetilde{n}(\xi)=\sum_{i=1}^{P} w_{i} \delta_{\sigma}^{(L)}\left(\xi, \xi_{i}\right)$ are given by

$$
\widetilde{m}_{k}=m_{k}^{*} z^{k^{2}}
$$

This can be translated into a linear system

$$
\widetilde{\boldsymbol{m}}_{n}=\boldsymbol{A}_{n}^{(L)}(\sigma) \cdot \boldsymbol{m}_{n}^{*}
$$

with $\boldsymbol{A}_{n}^{(L)}(\sigma)$ a diagonal matrix:

$$
A_{i, j}^{(L)}(\sigma)= \begin{cases}z^{i^{2}} & \text { if } i=j \\ 0 & \text { otherwise }\end{cases}
$$

whose inverse matrix is directly given by

$$
A_{i, j}^{(L)-1}(\sigma)= \begin{cases}z^{-i^{2}} & \text { if } i=j \\ 0 & \text { otherwise }\end{cases}
$$

\section{Appendix B.3.3. Low cost nested quadrature}

A variable change allows approximating integral properties over a LogN EQMOM reconstruction using Gauss-Hermite quadratures [18]:

$$
\int_{0}^{+\infty} f(\xi) n(\xi) \mathrm{d} \xi \approx \frac{1}{\sqrt{\pi}} \sum_{i=1}^{P} w_{i} \sum_{j=1}^{Q} \omega_{j} f\left(\xi_{i} \exp \left(\sigma \lambda_{j} \sqrt{2}\right)\right)
$$

with $\boldsymbol{w}_{P}, \boldsymbol{\xi}_{P}$ and $\sigma$ the EQMOM reconstruction parameters computed from $\boldsymbol{m}_{2 P} ; \boldsymbol{\omega}_{Q}$ and $\boldsymbol{\lambda}_{Q}$ are the weights and nodes of a $Q$-nodes Gauss-Hermite quadrature rule (see Appendix C).

Parameters of this nested quadrature do not depend on $\sigma$ of the main quadrature nodes $\boldsymbol{\xi}_{P}$. Consequently, $\boldsymbol{\omega}_{Q}$ and $\boldsymbol{\lambda}_{Q}$ only need to be computed once. It is worth noting that this quadrature does not preserve the moments of the distribution and only yields exact results for $f(\xi)=\log (\xi)^{k}, k \in\{0, \ldots, 2 \min (P, Q)-1\}$.

Appendix B.3.4. Moment preserving nested quadrature

[16] suggested the use of Gauss-Wigert quadratures [33] to preserve the moments of a 
LogN EQMOM reconstruction:

$$
\int_{0}^{+\infty} f(\xi) n(\xi) \mathrm{d} \xi \approx \sum_{i=1}^{P} w_{i} \sum_{j=1}^{Q} \omega_{j}^{(\sigma)} f\left(\xi_{i} \lambda_{j}^{(\sigma)}\right)
$$

with $\boldsymbol{w}_{P}, \boldsymbol{\xi}_{P}$ and $\sigma$ the EQMOM reconstruction parameters computed from $\boldsymbol{m}_{2 P} ; \boldsymbol{\omega}_{Q}^{(\sigma)}$ and $\boldsymbol{\lambda}_{Q}^{(\sigma)}$ are the weights and nodes of a $Q$-nodes Gauss-Wigert quadrature rule of parameter $\sigma$ (see Appendix C). This quadrature rule must be computed for each value of $\sigma$, i.e. for each LogN EQMOM reconstruction.

Appendix B.3.5. Single node analytical solution

The case $P=1$ has the following analytical solution:

$$
\begin{aligned}
w_{1} & =m_{0} \\
\xi_{1} & =\sqrt{\frac{m_{1}^{4}}{m_{2} m_{0}^{3}}} \\
\sigma & =\sqrt{\log \left(\frac{m_{2} m_{0}}{m_{1}^{2}}\right)}
\end{aligned}
$$

Appendix B.4. Gamma kernel

Appendix B.4.1. Definition

The Gamma kernel $\delta_{\sigma}^{(\Gamma)}\left(\xi, \xi_{m}\right)$ was first used in EQMOM by Yuan et al. [15]. It is defined on $\left.\Omega_{\xi}=\right] 0,+\infty[$ by

$$
\delta_{\sigma}^{(\Gamma)}\left(\xi, \xi_{m}\right)=\frac{\xi^{(l-1)} \exp (-\xi / \sigma)}{\Gamma(l) \sigma^{l}} \quad \text { with } l=\frac{\xi_{m}}{\sigma} \text { and } \Gamma(x)=\int_{0}^{+\infty} t^{x-1} e^{-t} \mathrm{~d} t
$$

Appendix B.4.2. Moments and linear system

Moments of the Gamma kernel are given by

$$
\int_{0}^{+\infty} \xi^{k} \delta_{\sigma}^{(\Gamma)}\left(\xi, \xi_{m}\right) \mathrm{d} \xi=G_{k}\left(\xi_{m}, \sigma\right)= \begin{cases}1 & \text { if } k=0 \\ \prod_{j=0}^{k-1}\left(\xi_{m}+j \sigma\right) & \text { otherwise }\end{cases}
$$

Moments of the distribution $\widetilde{n}(\xi)=\sum_{i=1}^{P} w_{i} \delta_{\sigma}^{(\Gamma)}\left(\xi, \xi_{i}\right)$ are given by the linear system

$$
\widetilde{\boldsymbol{m}}_{n}=\boldsymbol{A}_{n}^{(\Gamma)}(\sigma) \cdot \boldsymbol{m}_{n}^{*}
$$

with 


$$
A_{i, j}^{(\Gamma)}(\sigma)= \begin{cases}0 & \text { if } j>i \text { or } i=0 \text { or } j=0 \\ 1 & \text { if } i=0 \text { and } j=0 \\ A_{i-1, j-1}^{(\Gamma)}(\sigma)+(i-1) \sigma A_{i-1, j}^{(\Gamma)}(\sigma) & \text { otherwise }\end{cases}
$$

The inverse of this matrix is given by

$$
A_{i, j}^{(\Gamma)-1}(\sigma)= \begin{cases}0 & \text { if } j>i \text { or } i=0 \text { or } j=0 \\ 1 & \text { if } i=0 \text { and } j=0 \\ A_{i-1, j-1}^{(\Gamma)-1}(\sigma)-j \sigma A_{i-1, j}^{(\Gamma)-1}(\sigma) & \text { otherwise }\end{cases}
$$

which translates, for the case $N=6$ into

$$
\begin{gathered}
{\left[\begin{array}{c}
\widetilde{m}_{0} \\
\widetilde{m}_{1} \\
\widetilde{m}_{2} \\
\widetilde{m}_{3} \\
\widetilde{m}_{4} \\
\widetilde{m}_{5} \\
\widetilde{m}_{6}
\end{array}\right]=\left(\begin{array}{cccccccc}
1 & & & & & & 0 \\
0 & 1 & & & & & \\
0 & 1 \sigma & 1 & & & & \\
0 & 2 \sigma^{2} & 3 \sigma & 1 & & & \\
0 & 6 \sigma^{3} & 11 \sigma^{2} & 6 \sigma & 1 & & \\
0 & 24 \sigma^{4} & 50 \sigma^{3} & 35 \sigma^{2} & 10 \sigma & 1 & \\
0 & 120 \sigma^{5} & 274 \sigma^{4} & 225 \sigma^{3} & 85 \sigma^{2} & 15 \sigma & 1
\end{array}\right) \cdot\left[\begin{array}{c}
m_{0}^{*} \\
m_{1}^{*} \\
m_{2}^{*} \\
m_{3}^{*} \\
m_{4}^{*} \\
m_{5}^{*} \\
m_{6}^{*}
\end{array}\right]} \\
{\left[\begin{array}{c}
m_{0}^{*} \\
m_{1}^{*} \\
m_{3}^{*} \\
m_{4}^{*} \\
m_{5}^{*} \\
m_{6}^{*}
\end{array}\right]=\left(\begin{array}{cccccccc}
1 & & & & & & & 0 \\
0 & 1 & & & & & & \\
0 & -\sigma & 1 & & & & & \\
0 & \sigma^{2} & -3 \sigma & 1 & & & \\
0 & -\sigma^{3} & 7 \sigma^{2} & -6 \sigma & 1 & \\
0 & \sigma^{4} & -15 \sigma^{3} & 25 \sigma^{2} & -10 \sigma & 1 \\
0 & -\sigma^{5} & 31 \sigma^{4} & -90 \sigma^{3} & 65 \sigma^{2} & -15 \sigma & 1
\end{array}\right)\left[\begin{array}{c}
\widetilde{m}_{1} \\
\widetilde{m}_{2} \\
\widetilde{m}_{3} \\
\widetilde{m}_{4} \\
\widetilde{m}_{5} \\
\widetilde{m}_{6}
\end{array}\right]}
\end{gathered}
$$

\section{Appendix B.4.3. Low cost nested quadrature}

A Gauss-Laguerre quadrature can be used to approximate integral properties over a Gamma EQMOM reconstruction:

$$
\int_{0}^{+\infty} f(\xi) n(\xi) \mathrm{d} \xi \approx \sum_{j=1}^{Q} \omega_{j} f\left(\sigma \lambda_{j}\right) \sum_{i=1}^{P} \frac{w_{i}}{\Gamma\left(\frac{\xi_{i}}{\sigma}\right)} \lambda_{j}^{\frac{\xi_{i}}{\sigma}-1}
$$

with $\boldsymbol{w}_{P}, \boldsymbol{\xi}_{P}$ and $\sigma$ the EQMOM reconstruction parameters computed from $\boldsymbol{m}_{2 P} ; \boldsymbol{\omega}_{Q}$ and $\boldsymbol{\lambda}_{Q}$ are the weights and nodes of a $Q$-nodes Gauss-Laguerre quadrature rule of parameter $\alpha=0$ (see Appendix $\mathrm{C}$ ). The advantage of this quadrature is that it only requires $\boldsymbol{\omega}_{Q}$ and $\boldsymbol{\lambda}_{Q}$ to be computed once. However, this quadrature will not preserve the moments of the distribution.

\section{Appendix B.4.4. Moment preserving nested quadrature}

A generalized Gauss-Laguerre quadrature preserves the moments of a Gamma EQMOM reconstruction: 


$$
\int_{0}^{+\infty} f(\xi) n(\xi) \mathrm{d} \xi \approx \sum_{i=1}^{P} \frac{w_{i}}{\Gamma\left(\frac{\xi_{i}}{\sigma}\right)} \sum_{j=1}^{Q} \omega_{j}^{\left(\alpha_{i}\right)} f\left(\sigma \lambda_{j}^{\left(\alpha_{i}\right)}\right)
$$

with $\boldsymbol{w}_{P}, \boldsymbol{\xi}_{P}$ and $\sigma$ the EQMOM reconstruction parameters computed from $\boldsymbol{m}_{2 P} ; \boldsymbol{\omega}_{Q}^{\left(\alpha_{i}\right)}$ and $\boldsymbol{\lambda}_{Q}^{\left(\alpha_{i}\right)}$ are the weights and nodes of a $Q$-nodes Gauss-Laguerre quadrature rule of parameter $\alpha_{i}=\frac{\xi_{i}}{\sigma}-1$ (see Appendix C).

The accuracy of this quadrature comes with a cost related to the computation of $\boldsymbol{\omega}_{Q}^{\left(\alpha_{i}\right)}$ and $\boldsymbol{\lambda}_{Q}^{\left(\alpha_{i}\right)}$ for each value of $\alpha_{i}$.

\section{Appendix B.4.5. Single node analytical solution}

The case $P=1$ has the following analytical solution:

$$
\begin{aligned}
w_{1} & =m_{0} \\
\xi_{1} & =\frac{m_{1}}{m_{0}} \\
\sigma & =\frac{m_{2}}{m_{1}}-\frac{m_{1}}{m_{0}}
\end{aligned}
$$

Appendix B.5. Weibull kernel

Appendix B.5.1. Definition

The Weibull kernel $\delta_{\sigma}^{(W)}\left(\xi, \xi_{m}\right)$ is defined on $\left.\Omega_{\xi}=\right] 0,+\infty[$ by

$$
\delta_{\sigma}^{(W)}\left(\xi, \xi_{m}\right)=\frac{1}{\sigma \xi_{m}}\left(\frac{\xi}{\xi_{m}}\right)^{\frac{1-\sigma}{\sigma}} \exp \left(-\left(\frac{\xi}{\xi_{m}}\right)^{1 / \sigma}\right)
$$

Appendix B.5.2. Moments and linear system

Moments of the Weibull kernel are given by

$$
\int_{0}^{+\infty} \xi^{k} \delta_{\sigma}^{(W)}\left(\xi, \xi_{m}\right) \mathrm{d} \xi=\xi_{m}^{k} \Gamma(1+k \sigma)
$$

Moments of the distribution $\widetilde{n}(\xi)=\sum_{i=1}^{P} w_{i} \delta_{\sigma}^{(W)}\left(\xi, \xi_{i}\right)$ are given by

$$
\widetilde{m}_{k}=m_{k}^{*} \Gamma(1+k \sigma)
$$

This can be translated into a linear system

$$
\widetilde{\boldsymbol{m}}_{n}=\boldsymbol{A}_{n}^{(W)}(\sigma) \cdot \boldsymbol{m}_{n}^{*}
$$

with $\boldsymbol{A}_{n}^{(W)}(\sigma)$ a diagonal matrix:

$$
A_{i, j}^{(W)}(\sigma)= \begin{cases}\Gamma(1+i \sigma) & \text { if } i=j \\ 0 & \text { otherwise }\end{cases}
$$


whose inverse matrix is directly given by

$$
A_{i, j}^{(W)-1}(\sigma)= \begin{cases}\frac{1}{\Gamma(1+i \sigma)} & \text { if } i=j \\ 0 & \text { otherwise }\end{cases}
$$

Appendix B.5.3. Low cost nested quadrature

A Gauss-Laguerre quadrature can be used to approximate integral properties over a Weibull EQMOM reconstruction:

$$
\int_{0}^{+\infty} f(\xi) n(\xi) \mathrm{d} \xi \approx \sum_{i=1}^{P} w_{i} \sum_{j=1}^{Q} \omega_{j} f\left(\xi_{i} \lambda_{j}^{\sigma}\right)
$$

with $\boldsymbol{w}_{P}, \boldsymbol{\xi}_{P}$ and $\sigma$ the EQMOM reconstruction parameters computed from $\boldsymbol{m}_{2 P} ; \boldsymbol{\omega}_{Q}$ and $\boldsymbol{\lambda}_{Q}$ are the weights and nodes of a $Q$-nodes Gauss-Laguerre quadrature rule of parameter $\alpha=0$ (see Appendix C). The advantage of this quadrature is that it only requires $\boldsymbol{\omega}_{Q}$ and $\boldsymbol{\lambda}_{Q}$ to be computed once. However, this quadrature will not preserve the moments of the distribution and only yields exact results for $f(\xi)=\xi^{k / \sigma}, k \in\{0, \ldots, 2 \min (P, Q)-1\}$

\section{Appendix B.5.4. Moment preserving nested quadrature}

One can produce a Gauss quadrature that preserves the moments of Weibull EQMOM approximations:

$$
\int_{0}^{+\infty} f(\xi) n(\xi) \mathrm{d} \xi \approx \sum_{i=1}^{P} w_{i} \sum_{j=1}^{Q} \omega_{j}^{(\sigma)} f\left(\xi_{i} \lambda_{j}^{(\sigma)}\right)
$$

with $\boldsymbol{w}_{P}, \boldsymbol{\xi}_{P}$ and $\sigma$ the EQMOM reconstruction parameters computed from $\boldsymbol{m}_{2 P} ; \boldsymbol{\omega}_{Q}^{(\sigma)}$ and $\boldsymbol{\lambda}_{Q}^{(\sigma)}$ are the weights and nodes of a $Q$-nodes "Gauss-Weibull" quadrature rule of parameter $\sigma$ (see Appendix C). The weights and nodes of the nested quadrature need to be computed for each value of $\sigma$, i.e. for each Weibull EQMOM approximation of the NDF.

\section{Appendix B.5.5. Single node numerical solution}

The parameters $w_{1}, \xi_{1}$ and $\sigma$ of the one-node Weibull EQMOM must be solution of the following system:

$$
\begin{aligned}
m_{0} & =w_{1} \\
\frac{m_{1}}{\Gamma(1+\sigma)} & =w_{1} \xi_{1} \\
\frac{m_{2}}{\Gamma(1+2 \sigma)} & =w_{1} \xi_{1}^{2}
\end{aligned}
$$

The first equation gives $w_{1}=m_{0}$ but no explicit solution exists for the two other equations. One can however notice that $s=\frac{\sigma}{1+\sigma}$ must be a root of

$$
G(s)=\frac{m_{2} m_{0}}{m_{1}^{2}}-\frac{\Gamma\left(\frac{1+s}{1-s}\right)}{\Gamma\left(\frac{1}{1-s}\right)^{2}}
$$


which is monotonous, defined on $s \in[0,1[$ and has the following limits

$$
\begin{aligned}
G(0) & =\frac{m_{2} m_{0}}{m_{1}^{2}}>0 \\
\lim _{s \rightarrow 1^{-}} G(s) & <0
\end{aligned}
$$

$G(s)$ then admits a single root that can be computed numerically with the Ridder's method. One can also narrow down, at a very low cost, the search interval [0, 1[ by using the property

$$
g_{n}=G\left(\frac{n}{n+1}\right)=\frac{m_{2} m_{0}}{m_{1}^{2}}-\frac{(2 n) !}{(n !)^{2}}
$$

with $n$ an integer, which induces the following recurrence relation:

$$
\begin{aligned}
g_{n} & =c-h_{n} \\
h_{n+1} & =\left(4-\frac{2}{n+1}\right) h_{n}
\end{aligned}
$$

with $c=\frac{m_{2} m_{0}}{m_{1}^{2}}$ and $h_{1}=2$.

The proposed algorithm to identify the root of $G(s)$ is

1. Compute $c=\frac{m_{2} m_{0}}{m_{1}^{2}}$

- if $c<1$, cancel the operation as the moments are not realisable;

- if $c=1, s=0$ is the root of $G(s)$;

- if $c<2$, set $s_{l}=0, v_{l}=c-1, s_{r}=\frac{1}{2}$ and $v_{r}=c-2$ and go to step 3 .

- otherwise, set $s_{l}=0, v_{l}=c-1$ and go to step 2 .

2. Initialise $i=1, h=2$ and iterate

(a) increment $i$ by 1 ;

(b) compute $h=h *\left(4-\frac{2}{i}\right)$

- if $h=c$, then $s=\frac{i}{i+1}$ is a root of $G(s)$;

- if $h<c$, set $s_{l}=\frac{i}{i+1}$ and $v_{l}=c-h$;

- if $h>c$, set $s_{r}=\frac{i}{i+1}, v_{r}=c-h$ and go to step 3 .

3. Apply the Ridder's method to $G(s)$ on the interval $\left[s_{l}, s_{r}\right]$

(a) compute $s_{t_{1}}=\frac{1}{2}\left(s_{l}+s_{r}\right)$ and $v_{t_{1}}=G\left(s_{t_{1}}\right)$;

(b) compute $s_{t_{2}}=s_{t_{1}}+\left(s_{t_{1}}-s_{l}\right) \frac{v_{t_{1}}}{\sqrt{v_{t_{1}}^{2}-v_{l} v_{r}}}$ and $v_{t_{2}}=G\left(s_{t_{2}}\right)$;

(c) set $s_{l}$ the highest value between $s_{l}, s_{t_{1}}$ and $s_{t_{2}}$ whose image by $G$ is positive;

(d) set $s_{r}$ the lowest value between $s_{r}, s_{t_{1}}$ and $s_{t_{2}}$ whose image by $G$ is negative;

(e) stop the computation if $v_{l}<\varepsilon(c-1)$ with $\varepsilon$ a relative tolerance $\left(\right.$ e.g. $\left.\varepsilon=10^{-10}\right)$ and consider $s_{l}$ as a root of $G(s)$. 
Once the root of $G(s)$ is identified, compute

$$
\begin{aligned}
\sigma & =\frac{s}{1-s} \\
\xi_{1} & =\frac{m_{1}}{m_{0} \Gamma(1+\sigma)}
\end{aligned}
$$

Note that each iteration of the Ridder's method requires two computations of $G(s)$, that implies four computations of the Gamma function -which is quite expensive- by iteration. This explains the interest of the second step which allows to narrow down the research interval at hardly no cost.

\section{Appendix B.6. Beta kernel}

\section{Appendix B.6.1. Definition}

The Beta kernel $\delta_{\sigma}^{(\beta)}\left(\xi, \xi_{m}\right)$ was first used in EQMOM by Yuan et al. [15]. It is defined on $\left.\Omega_{\xi}=\right] 0,1[$ by

$$
\delta_{\sigma}^{(\beta)}\left(\xi, \xi_{m}\right)=\frac{\xi^{(l-1)}(1-\xi)^{(m-1)}}{B(l, m)} \quad \text { with } l=\frac{\xi_{m}}{\sigma} \text { and } m=\frac{1-\xi_{m}}{\sigma}
$$

with $B(l, m)=\int_{0}^{1} x^{(l-1)}(1-x)^{(m-1)} \mathrm{d} x$ the beta function.

\section{Appendix B.6.2. Moments and linear system}

Moments of the Beta kernel are given by

$$
\int_{0}^{1} \xi^{k} \delta_{\sigma}^{(\beta)}\left(\xi, \xi_{m}\right) \mathrm{d} \xi=H_{k}\left(\xi_{m}, \sigma\right)= \begin{cases}1 & \text { if } k=0 \\ \prod_{j=0}^{k-1}\left(\frac{\xi_{m}+j \sigma}{1+j \sigma}\right) & \text { otherwise }\end{cases}
$$

Moments of the distribution $\widetilde{n}(\xi)=\sum_{i=1}^{P} w_{i} \delta_{\sigma}^{(\beta)}\left(\xi, \xi_{i}\right)$ are given by the linear system

$$
\widetilde{\boldsymbol{m}}_{n}=\boldsymbol{A}_{n}^{(\beta)}(\sigma) \cdot \boldsymbol{m}_{n}^{*}
$$

with the elements of $\boldsymbol{A}_{n}^{(\beta)}(\sigma)$ being computed from the elements of the matrix relative to Gamma EQMOM, $\boldsymbol{A}_{n}^{(\Gamma)}(\sigma)$ :

$$
\begin{aligned}
A_{i, j}^{(\beta)}(\sigma) & =\frac{A_{i, j}^{(\Gamma)}(\sigma)}{F_{i}(\sigma)} \\
F_{i}(\sigma) & = \begin{cases}1 & \text { if } i \leq 1 \\
(1+(i-1) \sigma) F_{i-1}(\sigma) & \text { otherwise }\end{cases}
\end{aligned}
$$

The inverse of this matrix is also easily defined from $\boldsymbol{A}_{n}^{(\Gamma)-1}(\sigma)$ :

$$
A_{i, j}^{(\beta)-1}(\sigma)=A_{i, j}^{(\Gamma)-1}(\sigma) F_{j}(\sigma)
$$


Appendix B.6.3. Low cost nested quadrature

A Gauss-Legendre quadrature can be used to approximate integral properties over a Beta EQMOM reconstruction:

$$
\int_{0}^{1} f(\xi) n(\xi) \mathrm{d} \xi \approx \frac{1}{2} \sum_{i=1}^{P} \frac{w_{i}}{B\left(\alpha_{i}+1, \beta_{i}+1\right)} \sum_{j=1}^{Q} \omega_{j} f\left(\frac{1-\lambda_{j}}{2}\right)\left(\frac{1-\lambda_{j}}{2}\right)^{\alpha_{i}}\left(\frac{1+\lambda_{j}}{2}\right)^{\beta_{i}}
$$

with $\boldsymbol{w}_{P}, \boldsymbol{\xi}_{P}$ and $\sigma$ the EQMOM reconstruction parameters computed from $\boldsymbol{m}_{2 P} ; \boldsymbol{\omega}_{Q}$ and $\boldsymbol{\lambda}_{Q}$ are the weights and nodes of a $Q$-nodes Gauss-Legendre quadrature rule (see Appendix C); $\alpha_{i}=\frac{\xi_{i}-\sigma}{\sigma}$ and $\beta_{i}=\frac{1-\xi_{i}-\sigma}{\sigma}$. This nested quadrature only requires $\boldsymbol{\omega}_{Q}$ and $\boldsymbol{\lambda}_{Q}$ to be computed once, but will not preserve the moments of the distribution.

\section{Appendix B.6.4. Moment preserving nested quadrature}

A Gauss-Jacobi quadrature will preserve the moments of the distribution:

$$
\int_{0}^{1} f(\xi) n(\xi) \mathrm{d} \xi \approx 2^{\frac{\sigma-1}{\sigma}} \sum_{i=1}^{P} \frac{w_{i}}{B\left(\alpha_{i}+1, \beta_{i}+1\right)} \sum_{j=1}^{Q} \omega_{j}^{\left(\alpha_{i}, \beta_{i}\right)} f\left(\frac{1-\lambda_{j}^{\left(\alpha_{i}, \beta_{i}\right)}}{2}\right)
$$

with $\boldsymbol{w}_{P}, \boldsymbol{\xi}_{P}$ and $\sigma$ the EQMOM reconstruction parameters computed from $\boldsymbol{m}_{2 P} ; \boldsymbol{\omega}_{Q}^{\left(\alpha_{i}, \beta_{i}\right)}$ and $\boldsymbol{\lambda}_{Q}^{\left(\alpha_{i}, \beta_{i}\right)}$ are the weights and nodes of a $Q$-nodes Gauss-Jacobi quadrature rule of parameters $\alpha_{i}=\frac{\xi_{i}-\sigma}{\sigma}$ and $\beta_{i}=\frac{1-\xi_{i}-\sigma}{\sigma}$ (see Appendix $\mathrm{C}$ ). The moment-preserving property of this quadrature comes with the need to compute $\boldsymbol{\omega}_{Q}^{\left(\alpha_{i}, \beta_{i}\right)}$ and $\boldsymbol{\lambda}_{Q}^{\left(\alpha_{i}, \beta_{i}\right)}$ for each node of the main Beta EQMOM quadrature.

Appendix B.6.5. Single node analytical solution

The case $P=1$ has the following analytical solution:

$$
\begin{aligned}
w_{1} & =m_{0} \\
\xi_{1} & =\frac{m_{1}}{m_{0}} \\
\sigma & =\frac{m_{1}^{2}-m_{0} m_{2}}{m_{0}\left(m_{2}-m_{1}\right)}
\end{aligned}
$$

\section{Appendix C. Gaussian quadratures}

A Q-node Gaussian quadrature allows to approximate a function integral as a weighted sum of pointwise values of this function over an interval $I$ :

$$
\int_{I} f(x) p(x) \mathrm{d} x \approx \sum_{j=1}^{Q} \omega_{j} f\left(\lambda_{j}\right)
$$


$p(x)$ is a weight function, and the quadrature rule yields accurate integral evaluations if $f(x)=x^{k}, k \in\{0, \ldots, 2 Q-1\}$. The computation of the weights $\boldsymbol{\omega}_{Q}$ and nodes $\boldsymbol{\lambda}_{Q}$ is performed as detailed in 2.2 by considering polynomials that are orthogonal with respect to the weight function $p(x)$.

Table C.1 details for each Gauss quadrature:

- the weight function $p(x)$;

- the integration support $I$;

- the computation of recurrence coefficients $\boldsymbol{a}_{Q-1}$ and $\boldsymbol{b}_{Q-1}$;

- the zero-th order moment $P_{0}$ of $p(x)$.

The recurrence coefficients are used to construct the Jacobi matrix $\boldsymbol{J}_{Q}$ associated with $p(x)$ on $I$ (see Eq. 6). The nodes $\boldsymbol{\lambda}_{Q}$ are the eigenvalues of $\boldsymbol{J}_{Q}$, and the weights $\boldsymbol{\omega}_{Q}$ are given by $\omega_{j}=P_{0} v_{1, j}^{2}$ with $v_{1, j}$ the first component of the normalised eigenvector belonging to the eigenvalue $\lambda_{j}$. 
Table C.1: Specifics of Gauss quadratures used for EQMOM nested quadratures.

\begin{tabular}{|c|c|c|c|c|}
\hline Gauss- & $I$ & $p(x)$ & $\boldsymbol{a}_{Q}$ and $\boldsymbol{b}_{Q}$ & $P_{0}$ \\
\hline Hermite & $\mathbb{R}$ & $\exp \left(-x^{2}\right)$ & $\begin{aligned} a_{k} & =0 \\
b_{k} & =k / 2\end{aligned}$ & $\sqrt{\pi}$ \\
\hline Laplace $^{\mathrm{c}}$ & $\mathbb{R}$ & $\exp (-|x|) / 2$ & $\begin{array}{l}\text { Apply Chebyshev algorithm to } \\
\boldsymbol{P}_{2 Q-1} \text { with } P_{k}= \begin{cases}0 & \text { if } k \text { odd } \\
k ! & \text { if } k \text { even }\end{cases} \end{array}$ & 1 \\
\hline Laguerref $^{f}$ & $\mathbb{R}^{+}$ & $x^{\alpha} \exp (-x)$ & $\begin{array}{l}a_{0}=1+\alpha \\
a_{k}=2+a_{k-1} \\
b_{k}=k(k+\alpha)\end{array}$ & $\Gamma(1+\alpha)^{\mathrm{d}}$ \\
\hline Wigert ${ }^{\mathrm{a}, \mathrm{f}}$ & $\mathbb{R}^{+}$ & $\frac{1}{\gamma x \sqrt{2 \pi}} \exp \left(\frac{\log ^{2}(x)}{2 \gamma^{2}}\right)$ & $\begin{aligned} a_{k} & =\left(\left(z^{2}+1\right) z^{2 k}-1\right) z^{2 k-1} \\
b_{k} & =\left(z^{2 k}-1\right) z^{6 k-4} \\
z & =\exp \left(\gamma^{2} / 2\right)\end{aligned}$ & 1 \\
\hline Weibull ${ }^{\mathrm{a}, \mathrm{f}}$ & $\mathbb{R}^{+}$ & $\gamma x^{\gamma-1} \exp \left(-x^{\gamma}\right)$ & $\begin{array}{l}\text { Apply Chebyshev algorithm to } \\
\boldsymbol{P}_{2 Q-1} \text { with } P_{k}=\Gamma(1+k / \gamma)\end{array}$ & 1 \\
\hline Legendre $^{b}$ & ]$-1,1[$ & 1 & $\begin{aligned} a_{k} & =0 \\
b_{k} & =\frac{k^{2}}{4 k^{2}-1}\end{aligned}$ & 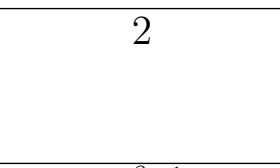 \\
\hline Jacobi $^{\mathrm{b}, \mathrm{f}}$ & ]$-1,1[$ & $(1-x)^{\alpha}(1+x)^{\beta}$ & $\begin{aligned} a_{k} & =\frac{\beta^{2}-\alpha^{2}}{\delta_{k}\left(\delta_{k}+2\right)} \\
b_{k} & =\frac{4 k(k+\alpha)(k+\beta)(k+\alpha+\beta)}{\delta_{k}^{2}\left(\delta_{k}^{2}-1\right)} \\
\delta_{k} & =2 k+\alpha+\beta\end{aligned}$ & $\begin{array}{c}2^{\alpha+\beta+1} \times \\
B(\alpha+1, \beta+1)^{\mathrm{e}}\end{array}$ \\
\hline
\end{tabular}

${ }^{\mathrm{a}}$ Wilck [33]. ${ }^{\mathrm{b}}$ Shen et al. [34]. ${ }^{\mathrm{c}}$ Not standard Gauss-quadrature. ${ }^{\mathrm{d}} \Gamma(x)=\int_{0}^{+\infty} t^{x-1} e^{-t} \mathrm{~d} t .{ }^{\mathrm{e}}$ $B(x, y)=\frac{\Gamma(x) \Gamma(y)}{\Gamma(x+y)} \cdot{ }^{\mathrm{f}} \alpha>-1, \beta>-1, \gamma>0$. 